\title{
ON THE MARTINGALE DECOMPOSITIONS OF GUNDY, MEYER, AND YOEURP IN INFINITE DIMENSIONS
}

\author{
IVAN YAROSLAVTSEV
}

\begin{abstract}
We show that the canonical decomposition (comprising both the Meyer-Yoeurp and the Yoeurp decompositions) of a general $X$-valued local martingale is possible if and only if $X$ has the UMD property. More precisely, $X$ is a UMD Banach space if and only if for any $X$-valued local martingale $M$ there exist a continuous local martingale $M^{c}$, a purely discontinuous quasi-left continuous local martingale $M^{q}$, and a purely discontinuous local martingale $M^{a}$ with accessible jumps such that $M=M^{c}+M^{q}+M^{a}$. The corresponding weak $L^{1}$-estimates are provided. Important tools used in the proof are a new version of Gundy's decomposition of continuous-time martingales and weak $L^{1}$-bounds for a certain class of vector-valued continuous-time martingale transforms.
\end{abstract}

\section{INTRODUCTION}

It is well-known thanks to the scalar-valued stochastic integration theory that a stochastic integral $\int \Phi \mathrm{d} N$ of a general bounded predictable real-valued process $\Phi$ with respect to a general real-valued local martingale $N$ exists and is well defined (see e.g. Chapter 26 in [26]). Moreover, $\int \Phi \mathrm{d} N$ is a local martingale, so by the Burkholder-Davis-Gundy inequalities one can show the corresponding $L^{p}$-estimates for $p \in(1, \infty)$ :

$$
\mathbb{E} \sup _{0 \leq s \leq t}\left|\int_{0}^{s} \Phi \mathrm{d} N\right|^{p} \bar{\sim}_{p} \mathbb{E}\left(\int_{0}^{t} \Phi^{2} \mathrm{~d}[N]\right)^{\frac{p}{2}}, \quad t \geq 0
$$

(here $[N]: \mathbb{R}_{+} \times \Omega \rightarrow \mathbb{R}_{+}$is a quadratic variation of $N$, see (4.3) for the definition). The inequality (1.1) together with a Banach fixed point argument play an important rôle in providing solutions to SPDE's with a general martingale noise (see e.g. [12, 20, 21, 26, 37, 49] and references therein). For this reason (1.1)-type inequalities for a broader class of $N$ and $\Phi$ are of interest. In particular, one can consider $H$-valued $N$ and $\mathcal{L}(H, X)$-valued $\Phi$ for some Hilbert space $H$ and Banach space $X$. Building on ideas of Garling [16] and McConnell [34], van Neerven, Veraar, and Weis have shown in [37] that for a special choice of $N$ (namely, $N$ being a Brownian motion) and a general process $\Phi$ it is necessary and sufficient that $X$ is in the class of so-called UMD Banach spaces (see Subsection 2.1 for the definition) in order to obtain estimates of the form (1.1) with the right-hand side replaces by a generalized square function. Later in the paper [48] by Veraar and in the paper

2010 Mathematics Subject Classification. 60G44 Secondary: 60G07, 60G57, 60H99, 46N30.

Key words and phrases. Gundy's decomposition, continuous-time martingales, UMD spaces, canonical decomposition, Meyer-Yoeurp decomposition, Yoeurp decomposition, weak estimates, weak differential subordination. 
[49] by Veraar and the author, inequalities of the form (1.1) have been extended to a general continuous martingale $N$, again given that $X$ has the UMD property.

Extending (1.1) to a general martingale $N$ is an open problem, which was solved only for $X=L^{q}(S)$ with $q \in(1, \infty)$ in the recent work [15] by Dirksen and the author. One of the key tools applied therein was the so-called canonical decomposition of martingales. The canonical decomposition first appeared in the work 54 by Yoeurp, and partly in the paper 36 by Meyer, and has the following form: an $X$-valued local martingale $M$ is said to admit the canonical decomposition if there exists a continuous local martingale $M^{c}$, a purely discontinuous quasi-left continuous local martingale $M^{q}$ (a "Poisson-like" martingale which does not jump at predictable stopping times), and a purely discontinuous local martingale $M^{a}$ with accessible jumps (a "discrete-like" martingale which jumps only at a certain countable set of predictable stopping times) such that $M_{0}^{c}=M_{0}^{q}=0$ a.s. and $M=M^{c}+M^{q}+M^{a}$. The canonical decomposition (if it exists) is unique due to the uniqueness in the case $X=\mathbb{R}$ (see Remark 4.2 and 4.4). Moreover, when $X$ is UMD one has by [51] that for all $p \in(1, \infty)$,

$$
\mathbb{E}\left\|M_{t}\right\|^{p} \bar{\sim}_{p, X} \mathbb{E}\left\|M_{t}^{c}\right\|^{p}+\mathbb{E}\left\|M_{t}^{q}\right\|^{p}+\mathbb{E}\left\|M_{t}^{a}\right\|^{p}, t \geq 0 .
$$

In particular, if $N$ is $H$-valued and $\Phi$ is $\mathcal{L}(H, X)$-valued, then

$$
\int \Phi \mathrm{d} N=\int \Phi \mathrm{d} N^{c}+\int \Phi \mathrm{d} N^{q}+\int \Phi \mathrm{d} N^{a}
$$

is the canonical decomposition given that $N=N^{c}+N^{q}+N^{a}$ is the canonical decomposition, so

$$
\mathbb{E}\left\|\int_{0}^{t} \Phi \mathrm{d} N\right\|^{p} \bar{\sim}_{p, X} \mathbb{E}\left\|\int_{0}^{t} \Phi \mathrm{d} N^{c}\right\|^{p}+\mathbb{E}\left\|\int_{0}^{t} \Phi \mathrm{d} N^{q}\right\|^{p}+\mathbb{E}\left\|\int_{0}^{t} \Phi \mathrm{d} N^{a}\right\|^{p}, \quad t \geq 0,
$$

which together with Doob's maximal inequality reduces the problem of extending (1.1) to the separate cases of $N^{c}, N^{q}$ and $N^{a}$. Possible approaches of how to work with $\int \Phi \mathrm{d} N^{c}, \int \Phi \mathrm{d} N^{q}$, and $\int \Phi \mathrm{d} N^{a}$ have been provided by [15]: sharp estimates for the first were already obtained in [48, 49] and follow from the similar estimates for a Brownian motion from [37]; the second can be treated by using random measure theory (see Subsection 2.4), which is an extension of Poisson random measure integration theory (see [13] and [14]); finally, the latter one can be transformed to a discrete martingale by an approximation argument, so the desired $L^{p}$-estimates are nothing more but the Burkholder-Rosenthal inequalities (see [5, 15, 46] for details).

The canonical decomposition also plays a significant rôle in obtaining $L^{p}$-estimates for weakly differentially subordinated martingales. The weak differential subordination property as a vector-valued generalization of Burkholder's differential subordination property (see [7, 23, 30, 40]) was introduced by the author in [53], and can be described in the following way: an $X$-valued local martingale $\widetilde{M}$ is weakly differentially subordinated to an $X$-valued local martingale $M$ if for each $x^{*} \in X^{*}$ and for each $t \geq s \geq 0$ a.s.

$$
\begin{aligned}
\left|\left\langle\widetilde{M}_{0}, x^{*}\right\rangle\right| & \leq\left|\left\langle M_{0}, x^{*}\right\rangle\right|, \\
{\left[\left\langle\widetilde{M}, x^{*}\right\rangle\right]_{t}-\left[\left\langle\widetilde{M}, x^{*}\right\rangle\right]_{s} } & \leq\left[\left\langle M, x^{*}\right\rangle\right]_{t}-\left[\left\langle M, x^{*}\right\rangle\right]_{s} .
\end{aligned}
$$

If $X$ is a UMD Banach space and $p \in(1, \infty)$, then applying $L^{p}$-bounds (1.2) for the terms of the canonical decomposition together with $L^{p}$-bounds for purely 
discontinuous (see [53]) and continuous (see [51]) weakly differentially subordinated martingales yields

$$
\left(\mathbb{E}\left\|\widetilde{M}_{\infty}\right\|^{p}\right)^{\frac{1}{p}} \leq c_{p, X}\left(\mathbb{E}\left\|M_{\infty}\right\|^{p}\right)^{\frac{1}{p}},
$$

where the best known constant $c_{p, X}$ equals $\beta_{p, X}^{2}\left(\beta_{p, X}+1\right)$ (here $\beta_{p, X}$ is the $U M D_{p}$ constant of $X$, see Subsection 2.1 for the definition). Sharp estimates for $c_{p, X}$ in (1.3) remain unknown. Moreover, it is an open problem whether one can prove weak $L^{1}$-estimates of the form

$$
\lambda \mathbb{P}\left(\widetilde{M}_{\infty}^{*}>\lambda\right) \lesssim_{p, X} \mathbb{E}\left\|M_{\infty}\right\|, \quad \lambda>0 .
$$

Here this question is partly solved: we show that (1.4) holds for $\widetilde{M}$ being one of the terms of the canonical decomposition of $M$ (see (1.5) and (4.2)).

The discussion above demonstrates that the canonical decomposition is useful for vector-valued stochastic integration and weak differential subordination, so the following natural question arises: for which Banach spaces $X$ does every $X$-valued local martingale have the canonical decomposition? The paper [51] together with the estimates (1.2) provides the answer for $L^{p}$-martingales given $p \in(1, \infty)$. Then $X$ being a UMD Banach space guarantees such a decomposition.

The present paper is devoted to providing the definitive answer to this question (see Section 4):

Theorem 1.1. Let $X$ be a Banach space. Then the following are equivalent:

(i) $X$ is a UMD Banach space;

(ii) every local martingale $M: \mathbb{R}_{+} \times \Omega \rightarrow X$ admits the canonical decomposition $M=M^{c}+M^{q}+M^{a}$.

Moreover, if this is the case, then for all $t \geq 0$ and $\lambda>0$

$$
\begin{aligned}
& \lambda \mathbb{P}\left(\left(M^{c}\right)_{t}^{*}>\lambda\right) \lesssim_{X} \mathbb{E}\left\|M_{t}\right\|, \\
& \lambda \mathbb{P}\left(\left(M^{q}\right)_{t}^{*}>\lambda\right) \lesssim_{X} \mathbb{E}\left\|M_{t}\right\|, \\
& \lambda \mathbb{P}\left(\left(M^{a}\right)_{t}^{*}>\lambda\right) \lesssim_{X} \mathbb{E}\left\|M_{t}\right\| .
\end{aligned}
$$

Notice that the inequalities (1.5) are new even in the real-valued case, even though in that case they are direct consequences of the sharp weak $(1,1)$-estimates for differentially subordinated martingales proven by Burkholder in [8, 9] (see also [39, 40] for details), from which one can show the following estimates

$$
\begin{aligned}
& \lambda \mathbb{P}\left(\left(M^{c}\right)_{t}^{*}>\lambda\right) \leq 2 \mathbb{E}\left|M_{t}\right|, \\
& \lambda \mathbb{P}\left(\left(M^{q}\right)_{t}^{*}>\lambda\right) \leq 2 \mathbb{E}\left|M_{t}\right|, \\
& \lambda \mathbb{P}\left(\left(M^{a}\right)_{t}^{*}>\lambda\right) \leq 2 \mathbb{E}\left|M_{t}\right| .
\end{aligned}
$$

The main instrument for proving $(i i) \Rightarrow(i)$ in Theorem 1.1 is Burkholder's characterization of UMD Banach spaces from [6]: $X$ is a UMD Banach space if and only if there exists a constant $C>0$ such that for any $X$-valued discrete martingale $\left(f_{n}\right)_{n \geq 0}$, for any sequence $\left(a_{n}\right)_{n \geq 0}$ with values in $\{-1,1\}$ one has that

$$
g_{\infty}^{*}>1 \text { a.s. } \Longrightarrow \mathbb{E}\left\|f_{\infty}\right\|>C,
$$

where $\left(g_{n}\right)_{n \geq 0}$ is an $X$-valued discrete martingale such that

$$
\begin{aligned}
g_{n}-g_{n-1} & =a_{n}\left(f_{n}-f_{n-1}\right), \quad n \geq 1, \\
g_{0} & =a_{0} f_{0},
\end{aligned}
$$


and where $g_{\infty}^{*}:=\sup _{n \geq 0}\left\|g_{n}\right\|$. Using this characterization for a given non-UMD Banach space $X$ we construct a martingale $M: \mathbb{R}_{+} \times \Omega \rightarrow X$ which does not have the canonical decomposition (see Subsection 4.4).

In order to obtain weak $L^{1}$-estimates of the form (1.5) together with $(i) \Rightarrow(i i)$ in Theorem 1.1 one needs to use two techniques. The first is the so-called Gundy decomposition of martingales. This decomposition was first obtained by Gundy in 19] for discrete real-valued martingales. Later in [11, 23, 33, 42] a more general version of this decomposition for vector-valued discrete martingales was obtained. In Section 3 we will present a continuous-time analogue of Gundy's decomposition, which has the following form: an $X$-valued martingale $M$ can be decomposed into a sum of three martingales $M^{1}, M^{2}$, and $M^{3}$, depending on $\lambda>0$, such that for each $t \geq 0$

(i) $\left\|M_{t}^{1}\right\|_{L^{\infty}(\Omega ; X)} \leq 2 \lambda, \mathbb{E}\left\|M_{t}^{1}\right\| \leq 5 \mathbb{E}\left\|M_{t}\right\|$,

(ii) $\lambda \mathbb{P}\left(\left(M^{2}\right)_{t}^{*}>0\right) \leq 4 \mathbb{E}\left\|M_{t}\right\|$,

(iii) $\mathbb{E}\left(\operatorname{Var} M^{3}\right)_{t} \leq 7 \mathbb{E}\left\|M_{t}\right\|$,

where $\operatorname{Var} M$ is a variation of the path of $M$.

The second important tool is weak differential subordination martingale transforms. Discrete martingale transforms were pioneered by Burkholder in [4], where he considered a transform $\left(f_{n}\right)_{n \geq 0} \mapsto\left(g_{n}\right)_{n \geq 0}$ of a real-valued martingale $\left(f_{n}\right)_{n \geq 0}$ such that

$$
\begin{aligned}
g_{n}-g_{n-1} & =a_{n}\left(f_{n}-f_{n-1}\right), \quad n \geq 1, \\
g_{0} & =a_{0} f_{0}
\end{aligned}
$$

for some $\{0,1\}$-valued deterministic sequence $\left(a_{n}\right)_{n \geq 0}$. Later in 6 , 11, 17, 22, 23, 33. several approaches and generalizations to the vector-valued setting and operatorvalued predictable sequence $\left(a_{n}\right)_{n \geq 0}$ have been discovered, while the martingale $\left(f_{n}\right)_{n>0}$ remained discrete. In particular for a very broad class of discrete martingale transforms it was shown that $L^{p}$-boundedness of the transform implies weak $L^{1}$ bounds. In Subsection 4.2 (see Theorem 4.9) we prove the same assertion for a weak differential subordination martingale transform, i.e. for an operator $T$ acting on continuous-time $X$-valued local martingales such that $T M$ is weakly differentially subordinated to $M$ and $\left\{M_{\infty}^{*}=0\right\} \subset\left\{(T M)_{\infty}^{*}=0\right\}$ for any $X$-valued local martingale $M$. A particular example of such a martingale transform $T$ is $M \mapsto$ $T M=M^{c}$, where $M^{c}$ is the continuous part of $M$ in the canonical decomposition. Due to (1.2) this operator is bounded as an operator acting on $L^{p}$-martingales if $X$ is UMD, so by Theorem 4.9 the first inequality of (1.5) follows. Even though in the case of a discrete filtration such an operator has a classical Burkholder's form (1.6) from [6] (with $\left(a_{n}\right)_{n \geq 0}$ being predictable instead of deterministic, see Proposition 4.13 and the remark thereafter), such transforms are of interest since they act on continuous-time martingales, which was not considered before.

Acknowledgment - The author would like to thank Mark Veraar for useful suggestions and fruitful discussions. The author thanks Alex Amenta and Jan van Neerven for careful reading of parts of this article and helpful comments.

\section{Preliminaries}

In the sequel the scalar field is assumed to be $\mathbb{R}$, unless stated otherwise. 
2.1. UMD Banach spaces. A Banach space $X$ is called a UMD space if for some (or equivalently, for all) $p \in(1, \infty)$ there exists a constant $\beta>0$ such that for every $N \geq 1$, every martingale difference sequence $\left(d_{n}\right)_{n=1}^{N}$ in $L^{p}(\Omega ; X)$, and every scalar-valued sequence $\left(\varepsilon_{n}\right)_{n=1}^{N}$ such that $\left|\varepsilon_{n}\right|=1$ for each $n=1, \ldots, N$ we have

$$
\left(\mathbb{E}\left\|\sum_{n=1}^{N} \varepsilon_{n} d_{n}\right\|^{p}\right)^{\frac{1}{p}} \leq \beta\left(\mathbb{E}\left\|\sum_{n=1}^{N} d_{n}\right\|^{p}\right)^{\frac{1}{p}} .
$$

The least admissible constant $\beta$ is denoted by $\beta_{p, X}$ and is called the $U M D_{p}$ constant or, if the value of $p$ is understood, the UMD constant, of $X$. It is well-known that UMD spaces obtain a large number of useful properties, such as being reflexive. Examples of UMD spaces include all finite dimensional spaces and the reflexive range of $L^{q}$-spaces, Besov spaces, Sobolev spaces, Schatten class spaces, and Orlicz spaces. Example of spaces without the UMD property include all nonreflexive Banach spaces, e.g. $L^{1}(0,1)$ and $C([0,1])$. We refer the reader to [10, 23, 43, 47] for details.

2.2. Martingales and càdlàg processes. Let $X$ be a Banach space, $\mathbb{F}=\left(\mathcal{F}_{t}\right)_{t \geq 0}$ be a filtration that satisfies the usual conditions (e.g. right-continuity). For each $1 \leq p<\infty$ a martingale $M: \mathbb{R}_{+} \times \Omega \rightarrow X$ is called an $L^{p}$-martingale (or, an $L^{p}$-integrable martingale) if $M_{\infty}:=\lim _{t \rightarrow \infty} M_{t}$ exists in $L^{p}(\Omega ; X)$; we call $M$ an $L^{\infty}$-martingale if $\left\|M_{t}\right\|_{L^{\infty}(\Omega ; X)}$ is uniformly bounded in $t \in \mathbb{R}_{+}$. For a given $p \in[1, \infty)$ we will denote the set of all $X$-valued $L^{p}$-integrable $\mathbb{F}$-martingales by $\mathcal{M}_{X}^{p}(\mathbb{F})$; further, we will denote the set of all $X$-valued local $L^{p}$-integrable $\mathbb{F}$-martingales by $\mathcal{M}_{X}^{p \text {,loc }}(\mathbb{F})$. Note that $\mathcal{M}_{X}^{p}(\mathbb{F})$ is a Banach space endowed with the norm $\|M\|_{\mathcal{M}_{X}^{p}(\mathbb{F})}:=\left\|M_{\infty}\right\|_{L^{p}(\Omega ; X)}$.

We will denote by $\mathcal{M}_{X}^{1, \infty}(\mathbb{F})$ the set of all $X$-valued local $\mathbb{F}$-martingales $M$ such that

$$
\sup _{\lambda>0} \lambda \mathbb{P}\left(M_{\infty}^{*}>\lambda\right)<\infty .
$$

In the sequel we will omit $\mathbb{F}$ from the notations $\mathcal{M}_{X}^{p}(\mathbb{F}), \mathcal{M}_{X}^{p, \text { loc }}(\mathbb{F})$, and $\mathcal{M}_{X}^{1, \infty}(\mathbb{F})$.

Remark 2.1. Let $X$ be a Banach space, $M: \mathbb{R}_{+} \times \Omega \rightarrow X$ be a martingale. Then $\left(N_{t}\right)_{t \geq 0}:=\left(\left\|M_{t}\right\|\right)_{t \geq 0}$ is a submartingale by [26, Lemma 7.11] and the fact that $x \mapsto\|x\|$ is a convex function on $X$. Moreover, by [28, Theorem 1.3.8(i)] we have that for each $t \geq 0, p \geq 1$ and $\lambda>0$

$$
\mathbb{P}\left(M_{t}^{*}>\lambda\right) \leq \frac{\mathbb{E}\left\|M_{t}\right\|^{p}}{\lambda^{p}} .
$$

A function $f: \mathbb{R}_{+} \rightarrow X$ is called càdlàg (a French abbreviation of the phrase "continuous from right, limits from left") if it is right-continuous and if it has lefthand limits. A process $V: \mathbb{R}_{+} \times \Omega \rightarrow X$ is called càdlàg if it has càdlàg paths. For instance, any martingale $M: \mathbb{R}_{+} \times \Omega \rightarrow X$ has a càdlàg version given $\mathbb{F}$ satisfies the usual assumptions (see 53] for details in the vector-valued setting).

Let $\tau$ be a stopping time. If $V: \mathbb{R}_{+} \times \Omega \rightarrow X$ is càdlàg, then we can define $\Delta V_{\tau}: \Omega \rightarrow X$ in the following way:

$$
\Delta V_{\tau}= \begin{cases}V_{0}, & \tau=0, \\ V_{\tau}-\lim _{\varepsilon \rightarrow 0} V_{0 \vee(\tau-\varepsilon)}, & 0<\tau<\infty, \\ 0, & \tau=\infty,\end{cases}
$$


where $\lim _{\varepsilon \rightarrow 0} V_{0 \vee(\tau-\varepsilon)}$ exists since $V$ has paths with left-hand limits.

One can define the so-called ucp topology (uniform convergence on compact sets in probability) on the linear space of all càdlàg adapted $X$-valued processes; convergence in this topology can be characterized in the following way: a sequence $\left(V^{n}\right)_{n \geq 1}$ of càdlàg adapted $X$-valued processes converges to $V: \mathbb{R}_{+} \times \Omega \rightarrow X$ in the ucp topology if for any $t \geq 0$ and $K>0$ we have that

$$
\mathbb{P}\left(\sup _{0 \leq s \leq t}\left\|V_{s}-V_{s}^{n}\right\|>K\right) \rightarrow 0 \quad n \rightarrow \infty .
$$

Then the following proposition holds.

Proposition 2.2. The linear space of all càdlàg adapted $X$-valued processes endowed with the ucp topology is complete.

Proof. This is just the vector-valued analogue of [45, Theorem 62], for which one needs to apply the vector-valued variation of [44, Problem V.1].

We state without proof the following elementary but useful statement.

Lemma 2.3. Let $X$ be a Banach space, $\left(f_{n}\right)_{n \geq 1}, f$ be continuous $X$-valued functions on $[0,1]$ such that $f_{n} \rightarrow f$ in $C([0,1] ; X)$ as $n \rightarrow \infty$. Then the function $F:[0,1] \rightarrow \mathbb{R}_{+}$defined as follows

$$
F(t)=\sup _{n}\left\|f_{n}(t)\right\|, \quad t \in[0,1],
$$

is continuous.

2.3. Purely discontinuous martingales. Let $M: \mathbb{R}_{+} \times \Omega \rightarrow \mathbb{R}$ be a local martingale. Then $M$ is called purely discontinuous if $[M]$ is a pure jump process (i.e. $[M]$ has a version that is a constant a.s. in time). Let $X$ be a Banach space, $M: \mathbb{R}_{+} \times \Omega \rightarrow X$ be a local martingale. Then $M$ is called purely discontinuous if for each $x^{*} \in X^{*}$ a local martingale $\left\langle M, x^{*}\right\rangle$ is purely discontinuous. The following proposition can be found in [15, 51].

Proposition 2.4. A martingale $M: \mathbb{R}_{+} \times \Omega \rightarrow X$ is purely discontinuous if and only if $M N$ is a martingale for any continuous bounded martingale $N: \mathbb{R}_{+} \times \Omega \rightarrow \mathbb{R}$ such that $N_{0}=0$.

In the sequel we will use the following lemma, which proof can be found in 15, 51.

Lemma 2.5. Let $X$ be a Banach space, $M: \mathbb{R}_{+} \times \Omega \rightarrow X$ be a martingale which is both continuous and purely discontinuous. Then $M=M_{0}$ a.s.

The reader can find more on purely discontinuous martingales in 15, 24, 25, 26, 51, 53].

2.4. Random measures. Let $(J, \mathcal{J})$ be a measurable space. Then a family $\mu=$ $\{\mu(\omega ; \mathrm{d} t, \mathrm{~d} x), \omega \in \Omega\}$ of nonnegative measures on $\left(\mathbb{R}_{+} \times J ; \mathcal{B}\left(\mathbb{R}_{+}\right) \otimes \mathcal{J}\right)$ is called a random measure. A random measure $\mu$ is called integer-valued if it takes values in $\mathbb{N} \cup\{\infty\}$, i.e. for each $A \in \mathcal{B}\left(\mathbb{R}_{+}\right) \otimes \mathcal{F} \otimes \mathcal{J}$ one has that $\mu(A) \in \mathbb{N} \cup\{\infty\}$ a.s., and if $\mu(\{t\} \times J) \in\{0,1\}$ a.s. for all $t \geq 0$.

Recall that $\mathcal{P}$ and $\mathcal{O}$ denote the predictable and optional $\sigma$-algebras on $\mathbb{R}_{+} \times \Omega$ and $\widetilde{\mathcal{P}}=\mathcal{P} \otimes \mathcal{J}$ and $\widetilde{\mathcal{O}}:=\mathcal{O} \otimes \mathcal{J}$ are the induced $\sigma$-algebras on $\widetilde{\Omega}:=\mathbb{R}_{+} \times \Omega \times J$. A process $F: \mathbb{R}_{+} \times \Omega \rightarrow \mathbb{R}$ is called optional if it is $\mathcal{O}$-measurable. A random 
measure $\mu$ is called optional (resp. predictable) if for any $\widetilde{\mathcal{O}}$-measurable (resp. $\widetilde{\mathcal{P}}$ measurable) nonnegative $F: \mathbb{R}_{+} \times \Omega \times J \rightarrow \mathbb{R}_{+}$the stochastic integral

$$
(t, \omega) \mapsto \int_{\mathbb{R}_{+} \times J} \mathbf{1}_{[0, t]}(s) F(s, \omega, x) \mu(\omega ; \mathrm{d} s, \mathrm{~d} x), \quad t \geq 0, \omega \in \Omega,
$$

as a function from $\mathbb{R}_{+} \times \Omega$ to $\overline{\mathbb{R}}_{+}$is optional (resp. predictable).

Let $X$ be a Banach space. Then we can extend stochastic integration to $X$-valued processes in the following way. Let $F: \mathbb{R}_{+} \times \Omega \times J \rightarrow X, \mu$ be a random measure. The integral

$$
t \mapsto \int_{\mathbb{R}_{+} \times J} F(s, \cdot, x) \mathbf{1}_{[0, t]}(s) \mu(\cdot ; \mathrm{d} s, \mathrm{~d} x), \quad t \geq 0,
$$

is well-defined and optional (resp. predictable) if $\mu$ is optional (resp. predictable), $F$ is $\widetilde{\mathcal{O}}$-strongly-measurable (resp. $\widetilde{\mathcal{P}}$-strongly-measurable), and $\int_{\mathbb{R}_{+} \times J}\|F\| \mathrm{d} \mu$ is a.s. bounded.

A random measure $\mu$ is called $\widetilde{\mathcal{P}}-\sigma$-finite if there exists an increasing sequence of sets $\left(A_{n}\right)_{n \geq 1} \subset \widetilde{\mathcal{P}}$ such that $\int_{\mathbb{R}_{+} \times J} \mathbf{1}_{A_{n}}(s, \omega, x) \mu(\omega ; \mathrm{d} s, \mathrm{~d} x)$ is finite a.s. and $\cup_{n} A_{n}=\mathbb{R}_{+} \times \Omega \times J$. According to [25, Theorem II.1.8] every $\widetilde{\mathcal{P}}-\sigma$-finite optional random measure $\mu$ has a compensator: a unique $\widetilde{\mathcal{P}}-\sigma$-finite predictable random measure $\nu$ such that $\mathbb{E} \int_{\mathbb{R}_{+} \times J} W \mathrm{~d} \mu=\mathbb{E} \int_{\mathbb{R}_{+} \times J} W \mathrm{~d} \nu$ for each $\widetilde{\mathcal{P}}$-measurable realvalued nonnegative $W$. We refer the reader to [25, Chapter II.1] for more details on random measures. For any optional $\widetilde{\mathcal{P}}-\sigma$-finite measure $\mu$ we define the associated compensated random measure by $\bar{\mu}=\mu-\nu$.

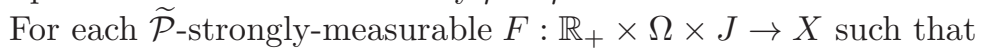

$$
\mathbb{E} \int_{\mathbb{R}_{+} \times J}\|F\| \mathrm{d} \mu<\infty
$$

(or, equivalently, $\mathbb{E} \int_{\mathbb{R}_{+} \times J}\|F\| \mathrm{d} \nu<\infty$, see the definition of a compensator above) we can define a process $t \mapsto \int_{[0, t] \times J}\|F\| \mathrm{d} \bar{\mu}$ by $\int_{[0, t] \times J} F \mathrm{~d} \mu-\int_{[0, t] \times J} F \mathrm{~d} \nu$. The following lemma is a vector-valued version of [25, Definition 1.27].

Lemma 2.6. Let $X$ be a Banach space, $\mu$ be a $\widetilde{\mathcal{P}}-\sigma$-finite optional random measure, $F: \mathbb{R}_{+} \times \Omega \times J \rightarrow X$ be $\widetilde{\mathcal{P}}$-strongly-measurable such that $\mathbb{E} \int_{\mathbb{R}_{+} \times J}\|F\| \mathrm{d} \mu<\infty$. Then $\left(\int_{[0, t] \times J} F \mathrm{~d} \bar{\mu}\right)_{t \geq 0}$ is a purely discontinuous $X$-valued martingale.

Proof. It is sufficient to show that

$$
t \mapsto\left\langle\int_{[0, t] \times J} F \mathrm{~d} \bar{\mu}, x^{*}\right\rangle=\int_{[0, t] \times J}\left\langle F, x^{*}\right\rangle \mathrm{d} \bar{\mu}, \quad t \geq 0,
$$

is a purely discontinuous martingale for each $x^{*} \in X^{*}$, which can be shown similarly the discussion right below [25, Definition 1.27]. 38].

The reader can find more information on random measures in [15, 25, 26, 31, 32,

2.5. Predictable and totally inaccessible stopping times. A stopping time $\tau$ is called predictable if there exists a sequence of stopping times $\left(\tau_{n}\right)_{n \geq 0}$ such that $\tau_{n}<\tau$ a.s. on $\{\tau>0\}$ and $\tau_{n} \nearrow \tau$ a.s. as $n \rightarrow \infty$. A stopping time $\tau$ is called totally inaccessible if $\mathbb{P}(\tau=\sigma)=0$ for any predictable stopping time $\sigma$. Later we will need the following lemma. 
Lemma 2.7. Let $X$ be a Banach space, $V: \mathbb{R}_{+} \times \Omega \rightarrow X$ be a predictable càdlàg process. Let $\tau$ be a totally inaccessible stopping time. Then $\Delta V_{\tau}=0$ a.s.

Proof. It is sufficient to show that $\left\langle\Delta V_{\tau}, x^{*}\right\rangle=0$ a.s. for any $x^{*} \in X^{*}$. Then the statement follows from [25, Proposition I.2.24].

Let $X$ be a Banach space, $M: \mathbb{R}_{+} \times \Omega \rightarrow X$ be a local martingale. Then $M$ has a càdlàg version (see e.g. [53]), and therefore we can define adapted càdlàg process $M^{\tau-}=\left(M_{t}^{\tau-}\right)_{t \geq 0}$ in the following way

$$
M_{t}^{\tau-}:=\lim _{\varepsilon \rightarrow 0} M_{(\tau-\varepsilon) \wedge t}, \quad t \geq 0,
$$

where we set $M_{t}=0$ for $t<0$. Notice that $M^{\tau-}$ is not necessarily a local martingale. For instance if $X=\mathbb{R}$ and $M$ is a compensated Poisson process, $\tau:=\inf _{t \geq 0}\left\{\Delta M_{t}>0\right\}$, then $M_{t}^{\tau-}=-(t \wedge \tau)$ a.s. for each $t \geq 0$, so it is a supermartingale which is not even a local martingale. Nevertheless, if $\tau$ is a predictable stopping time, then the following lemma holds. Recall that for any stopping time $\tau$ we define $\sigma$-field $\mathcal{F}_{\tau-}$ in the following way

$$
\mathcal{F}_{\tau-}:=\sigma\left\{\mathcal{F}_{0} \cup\left(\mathcal{F}_{t} \cap\{t<\tau\}\right), t>0\right\}
$$

(see [26, p. 491] for details).

Lemma 2.8. Let $X$ be a Banach space, $M: \mathbb{R}_{+} \times \Omega \rightarrow X$ be a local martingale, $\tau$ be a predictable stopping time. Then $M^{\tau-}$ defined as in (2.3) is a local martingale. Moreover, if $M$ is an $L^{1}$-martingale, then $M^{\tau-}$ is an $L^{1}$-martingale as well.

Proof. Without loss of generality we can let $M_{0}=0$ a.s. First assume that $M$ is an $L^{\infty}$-martingale. Let $\left(\tau_{n}\right)_{n \geq 1}$ be an announcing to $\tau$ sequence of stopping times, i.e. $\tau_{n}<\tau$ a.s. on $\{\tau>0\}$ and $\tau_{n} \nearrow \tau$ a.s. as $n \rightarrow \infty$. Then $M^{\tau_{n}}$ is an $L^{1}$-martingale for each $n \geq 1$. Moreover, $M_{t}^{\tau_{n}} \rightarrow M_{t}^{\tau-}$ a.s. as $n \rightarrow \infty$ for each $t \geq 0$. On the other hand, $M_{t}^{\tau_{n}}=\mathbb{E}\left(M_{t} \mid \mathcal{F}_{\tau_{n}}\right) \rightarrow \mathbb{E}\left(M_{t} \mid \mathcal{F}_{\tau-}\right)$ a.s. as $n \rightarrow \infty$ by [23, Theorem 3.3.8] and [26, Lemma 25.2(iii)], and hence in $L^{1}$ by the uniform boundedness due to the boundedness of $M_{\infty}$. Therefore for each $t \geq 0$ we have that $M_{t}^{\tau-}=\mathbb{E}\left(M_{t} \mid \mathcal{F}_{\tau_{-}}\right)$is integrable, hence for all $0 \leq s \leq t$

$$
\mathbb{E}\left(M_{t}^{\tau-} \mid \mathcal{F}_{s}\right)=\mathbb{E}\left(\lim _{n \rightarrow \infty} M_{t}^{\tau_{n}} \mid \mathcal{F}_{s}\right)=\lim _{n \rightarrow \infty} \mathbb{E}\left(M_{t}^{\tau_{n}} \mid \mathcal{F}_{s}\right)=\lim _{n \rightarrow \infty} M_{s}^{\tau_{n}}=M_{s}^{\tau-},
$$

where all the limits are taken in $L^{1}(\Omega ; X)$. Hence $\left(M_{t}^{\tau-}\right)_{t \geq 0}$ is a martingale. Moreover, by [23, Corollary 2.6.30]

$$
\mathbb{E}\left\|M_{t}^{\tau-}\right\|=\mathbb{E}\left\|\mathbb{E}\left(M_{t} \mid \mathcal{F}_{\tau-}\right)\right\| \leq \mathbb{E}\left\|M_{t}\right\| \leq \mathbb{E}\left\|M_{\infty}\right\|, \quad t \geq 0 .
$$

Now we treat the general case. Without loss of generality using a stopping time argument assume that $M$ is an $L^{1}$-martingale. Let $\left(M^{m}\right)_{m \geq 1}$ be a sequence of $X$-valued $L^{\infty}$-martingales such that $M_{\infty}^{m} \rightarrow M_{\infty}$ in $L^{1}(\Omega ; X)$ as $m \rightarrow \infty$. Analogously the first part of the proof $M_{t}^{\tau-}=\mathbb{E}\left(M_{t} \mid \mathcal{F}_{\tau-}\right)$ for each $t \geq 0$; moreover, by (2.4) $\left(\left(M^{m}\right)_{t}^{\tau-}\right)_{m \geq 1}$ is a Cauchy sequence in $L^{1}(\Omega ; X)$. Therefore by [23, Corollary 2.6.30], $\left(M^{m}\right)_{t}^{\tau-} \rightarrow M_{t}^{\tau-}$ in $L^{1}(\Omega ; X)$ for each $t \geq 0$, hence for each $t \geq s \geq 0$ by 23 , Corollary 2.6.30]

$$
\begin{aligned}
\mathbb{E}\left(M_{t}^{\tau-} \mid \mathcal{F}_{s}\right)=\mathbb{E}\left(\lim _{m \rightarrow \infty}\left(M^{m}\right)_{t}^{\tau-} \mid \mathcal{F}_{s}\right) & =\lim _{m \rightarrow \infty} \mathbb{E}\left(\left(M^{m}\right)_{t}^{\tau-} \mid \mathcal{F}_{s}\right) \\
& =\lim _{m \rightarrow \infty}\left(M^{m}\right)_{s}^{\tau-}=M_{s}^{\tau-},
\end{aligned}
$$


where all the limits are again taken in $L^{1}(\Omega ; X)$. Therefore $\left(M_{t}^{\tau-}\right)_{t \geq 0}$ is an $L^{1}$-martingale.

2.6. Compensator and variation. Let $X$ be a Banach space, $M: \mathbb{R}_{+} \times \Omega \rightarrow X$ be an adapted càdlàg process. Then a predictable process $V: \mathbb{R}_{+} \times \Omega$ is called a predictable compensator of $M$ (or just a compensator of $M$ ) if $V_{0}=0$ a.s. and if $M-V$ is a local martingale.

The variation $\operatorname{Var} M: \mathbb{R}_{+} \times \Omega \rightarrow \overline{\mathbb{R}}_{+}$of a càdlàg process $M: \mathbb{R}_{+} \times \Omega \rightarrow X$ is defined in the following way:

$$
(\operatorname{Var} M)_{t}:=\left\|M_{0}\right\|+\limsup _{\operatorname{mesh} \rightarrow 0} \sum_{n=1}^{N}\left\|M\left(t_{n}\right)-M\left(t_{n-1}\right)\right\|,
$$

where the limit superior is taken over all the partitions $0=t_{0}<\ldots<t_{N}=t$.

Let $V: \mathbb{R}_{+} \times \Omega \rightarrow X$ be a càdlàg adapted process. Analogously to the scalarvalued situation we can define a càdlàg adapted process $V^{*}: \mathbb{R}_{+} \times \Omega \rightarrow \mathbb{R}_{+}$of the following form

$$
V_{t}^{*}:=\sup _{s \in[0, t]}\left\|V_{s}\right\|, \quad t \geq 0
$$

\section{Gundy's DEComposition of COntinuous-time martingales}

For the proof of our main results, Theorem 4.8 and Theorem 4.9, we will need Gundy's decomposition of continuous-time martingales, which is a generalization of Gundy's decomposition of discrete martingales (see [19] and [23, Theorem 3.4.1] for the details).

Theorem 3.1 (Gundy's decomposition). Let $X$ be a Banach space, $M: \mathbb{R}_{+} \times$ $\Omega \rightarrow X$ be a martingale. Then for each $\lambda>0$ there exist martingales $M^{1}, M^{2}$ $M^{3}: \mathbb{R}_{+} \times \Omega \rightarrow X$ such that $M=M^{1}+M^{2}+M^{3}$ and

(i) $\left\|M_{t}^{1}\right\|_{L^{\infty}(\Omega ; X)} \leq 2 \lambda, \mathbb{E}\left\|M_{t}^{1}\right\| \leq 5 \mathbb{E}\left\|M_{t}\right\|$ for each $t \geq 0$,

(ii) $\lambda \mathbb{P}\left(\left(M^{2}\right)_{t}^{*}>0\right) \leq 4 \mathbb{E}\left\|M_{t}\right\|$ for each $t \geq 0$,

(iii) $\mathbb{E}\left(\operatorname{Var} M^{3}\right)_{t} \leq 7 \mathbb{E}\left\|M_{t}\right\|$ for each $t \geq 0$.

Remark 3.2. Notice that if $M$ is a discrete martingale (i.e. $M_{t}=M_{[t]}$ for any $t \geq 0$ ), then the decomposition in Theorem 3.1 turns to the classical discrete one from [23, Theorem 3.4.1].

For the proof we will need the following intermediate steps.

Lemma 3.3. Let $X$ be a Banach space, $M: \mathbb{R}_{+} \times \Omega \rightarrow X$ be a càdlàg adapted process such that $\mathbb{E}(\operatorname{Var} M)_{t}<\infty$ for each $t \geq 0$ and a.s.

$$
M_{t}=\sum_{0 \leq s \leq t} \Delta M_{s}, \quad t \geq 0 .
$$

Then $M$ has a càdlàg predictable compensator $V: \mathbb{R}_{+} \times \Omega \rightarrow X$ such that for each $t \geq 0$

$$
\mathbb{E}\left\|V_{t}\right\| \leq \mathbb{E}(\operatorname{Var} V)_{t} \leq \mathbb{E}(\operatorname{Var} M)_{t} .
$$

In particular, if $M$ has a.s. at most one jump, then

$$
\mathbb{E}\left\|V_{t}\right\| \leq \mathbb{E}(\operatorname{Var} V)_{t} \leq \mathbb{E}(\operatorname{Var} M)_{t}=\mathbb{E}\left\|M_{t}\right\| .
$$


Proof. Let $\mu^{M}$ be a random measure defined on $\mathbb{R}_{+} \times X$ pointwise in $\omega \in \Omega$ in the following way:

$$
\mu^{M}(\omega ; B \times A):=\sum_{u \in B} \mathbf{1}_{A \backslash\{0\}}\left(\Delta M_{u}(\omega)\right), \quad \omega \in \Omega, B \in \mathcal{B}\left(\mathbb{R}_{+}\right), A \in \mathcal{B}(X) .
$$

Notice that $(\operatorname{Var} M)_{t}=\sum_{0 \leq s \leq t}\left\|\Delta M_{s}\right\|$ a.s. for each $t \geq 0$, so in particular a.s.

$$
(\operatorname{Var} M)_{t}=\int_{[0, t] \times X}\|x\| \mathrm{d} \mu^{M}(x, s), \quad t \geq 0 .
$$

Also note that $\mu^{M}$ is $\mathcal{P}$ - $\sigma$-finite: for each $0 \leq u \leq v$ and $t \geq 0$ one has that

$$
\begin{aligned}
\mathbb{E} \int_{[0, t] \times X} \mathbf{1}_{\|x\| \in[u, v]} \mathrm{d} \mu^{M} & \bar{\sim}_{u, v} \mathbb{E} \int_{[0, t] \times X}\|x\| \mathbf{1}_{\|x\| \in[u, v]} \mathrm{d} \mu^{M} \\
& \leq \mathbb{E} \int_{[0, t] \times X}\|x\| \mathrm{d} \mu^{M} \\
& =\mathbb{E}(\operatorname{Var} M)_{t}<\infty .
\end{aligned}
$$

Since $\mu^{M}$ is an integer-valued optional $\mathcal{P}$ - $\sigma$-finite random measure, it has a predictable compensator $\nu^{M}$ (see Subsection 2.4 and [25, Theorem II.1.8]), and therefore since by (3.4)

$$
\mathbb{E} \int_{[0, t] \times X}\|x\| \mathrm{d} \mu^{M}(x, s)=\mathbb{E}(\operatorname{Var} M)_{t}<\infty,
$$

we have that

$$
t \mapsto V_{t}:=\int_{[0, t] \times X} x \mathrm{~d} \nu^{M}(x, s), \quad t \geq 0,
$$

is integrable and càdlàg in time due to the fact that it is an integral with respect to the measure $\nu^{M}$ a.s. Moreover, by the definition of variation (2.5) we have that $\left\|V_{t}\right\| \leq(\operatorname{Var} V)_{t}$ a.s. for each $t \geq 0$, and hence

$$
\begin{gathered}
\mathbb{E}\left\|V_{t}\right\| \leq \mathbb{E}(\operatorname{Var} V)_{t} \leq \mathbb{E} \int_{[0, t] \times X}\|x\| \mathrm{d} \nu^{M}(x, s) \stackrel{(*)}{=} \mathbb{E} \int_{[0, t] \times X}\|x\| \mathrm{d} \mu^{M}(x, s) \\
\stackrel{(* *)}{=} \mathbb{E}(\operatorname{Var} M)_{t},
\end{gathered}
$$

where $(*)$ holds due to the definition of a compensator, and $(* *)$ follows from (3.4). To show (3.2) it is sufficient to notice that if $M$ has at most one jump then $(\operatorname{Var} M)_{t}=\left\|M_{t}\right\|$ a.s. for each $t \geq 0$.

The following lemma is folklore, but the author could not find an appropriate reference, so we present it with the proof here.

Lemma 3.4. Let $X$ be a Banach space, $V: \mathbb{R}_{+} \times \Omega \rightarrow X$ be a right-continuous predictable process, $V_{0}=0$ a.s. Then $V$ is locally bounded.

Proof. For each $n \geq 0$ define a stopping time $\tau_{n}:=\inf \left\{t \geq 0:\left\|V_{t}\right\| \geq n\right\}$. Then a sequence $\left(\tau_{n}\right)_{n \geq 1}$ of stopping times is increasing a.s. and tends to infinity as $n \rightarrow \infty$. Moreover, $\left(\tau_{n}\right)_{n \geq 1}$ are predictable by [26, Theorem 25.14] and the fact that for each $n \geq 1$

$$
\{\tau \leq t\}=\left\{\sup _{0 \leq s \leq t}\left\|V_{s}\right\| \geq n\right\} \in \mathcal{P}
$$


Therefore for each $n \geq 1$ there exists an announcing sequence $\left(\tau_{m, n}\right)_{m \geq 1}$ of stopping times. Choose $m_{n}$ so that $\mathbb{P}\left(\tau_{n}-\tau_{m_{n}, n}>\frac{1}{2^{n}}\right)<\frac{1}{2^{n}}$. Then $\left(\tau_{m_{n}, n}\right)_{n \geq 1}$ is such that $\tau_{m_{n}, n} \rightarrow \infty$ a.s. as $n \rightarrow \infty$, and for each $n \geq 0$ we have that a.s. $\sup _{0 \leq s \leq \tau_{m_{n}, n}}\left\|V_{s}\right\| \leq \sup _{0 \leq s<\tau_{n}}\left\|V_{s}\right\| \leq n$.

Let $\tau$ and $\sigma$ be stopping times. Then we can set

$$
\tau-\wedge \sigma-:=(\tau \wedge \sigma)-\text {. }
$$

Notice that if $M: \mathbb{R}_{+} \times \Omega \rightarrow X$ is a càdlàg process, then $\left(M^{\tau-}\right)^{\sigma-}=M^{\tau-\wedge \sigma-}$.

Proof of Theorem 3.1. By a stopping time argument we can assume that $M$ is an $L^{1}$-martingale. Define a stopping time $\tau$ is the following way:

$$
\tau=\inf \left\{t \geq 0:\left\|M_{t}\right\| \geq \frac{\lambda}{2}\right\} .
$$

Let $M^{2,1}:=M-M^{\tau}$ and let $M^{3,1}(\cdot)=\Delta M_{\tau} \mathbf{1}_{[0, \cdot]}(\tau)+M_{0}^{\tau-}$, where by (2.3) we can conclude that a.s.

$$
M_{0}^{\tau-}:= \begin{cases}M_{0}, & \tau>0, \\ 0, & \tau=0 .\end{cases}
$$

Let $N: \mathbb{R}_{+} \times \Omega \rightarrow X$ be such that $N_{t}=\Delta M_{\tau} \mathbf{1}_{[0, t]}(\tau), t \geq 0$. Then due to the fact that $M_{\tau}=\mathbb{E}\left(M_{\infty} \mid \mathcal{F}_{\tau}\right)$ by [26, Theorem 7.29], 23, Corollary 2.6.30], and the fact that $\left\|M_{\tau-}\right\| \leq \frac{\lambda}{2}$ a.s., we get

$$
\begin{aligned}
\mathbb{E}(\operatorname{Var} N)_{\infty}=\mathbb{E}\left\|\Delta M_{\tau}\right\|=\mathbb{E}\left\|M_{\tau}-M_{\tau-}\right\| & \leq \mathbb{E}\left\|M_{\tau}\right\|+\mathbb{E}\left(\left\|M_{\tau-}\right\| \mathbf{1}_{\tau<\infty}\right) \\
& \leq \mathbb{E}\left\|M_{\infty}\right\|+\frac{\lambda}{2}<\infty .
\end{aligned}
$$

Therefore by Lemma $3.3 N$ has a compensator $V$. Let

$$
\sigma:=\inf \left\{t \geq 0:\left\|V_{t}\right\| \geq \lambda\right\}
$$

be a stopping time. Then by (3.5) $\sigma$ is a predictable stopping time. Define now $M^{1}=M^{\sigma-\wedge \tau-}+V^{\sigma-}-M_{0}^{\tau-}, M^{2,2}=\left(M^{\tau-}+V\right)-\left(M^{\sigma-\wedge \tau-}+V^{\sigma-}\right), M^{3,2}=$ $N-V$ where $\sigma-\wedge \tau-$ is defined as in (3.6). Define $M^{2}:=M^{2,1}+M^{2,2}$ and $M^{3}:=M^{3,1}+M^{3,2}$. Then $M=M^{1}+M^{2}+M^{3}$. Now let us describe why this is the right choice.

Step 1: $M^{1}$. First show that $M^{1}$ is a martingale. Indeed, for each $t \geq 0$

$$
\begin{aligned}
M_{t}^{1} & =M_{t}^{\sigma-\wedge \tau-}+V_{t}^{\sigma-}-M_{0}^{\tau-}=\left(M_{t}^{\tau-}+V_{t}-M_{0}^{\tau-}\right)^{\sigma-} \\
& =\left(M_{t}^{\tau-}-\mathbf{1}_{\tau \in[0, t]} \Delta M_{\tau}+V_{t}-M_{0}^{\tau_{2}-}\right)^{\sigma-} \\
& =\left(\left(M_{t}^{\tau-}-M_{0}^{\tau-}\right)-\left(N_{t}-V_{t}\right)\right)^{\sigma-},
\end{aligned}
$$

and the last expression is a martingale due to the fact that $M^{\tau}$ is a martingale by [26, Theorem 7.12], the fact that $N-V$ is a martingale by the definition of a compensator, Lemma 2.8, and the fact that by (3.8)

$$
\mathbb{E}\left\|N_{\infty}\right\| \leq \mathbb{E}(\operatorname{Var} N)_{\infty} \leq \mathbb{E}\left\|M_{\infty}\right\|+\frac{\lambda}{2}<\infty .
$$

Now let us check (i): $\left\|M_{\infty}^{\sigma-\wedge \tau-}\right\|,\left\|M_{0}^{\tau-}\right\| \leq \frac{\lambda}{2}$ a.s. by the definition of $\tau$, and $\left\|V_{\infty}^{\sigma-}\right\| \leq \lambda$ by the definition of $\sigma$, so $\left\|M_{\infty}^{1}\right\| \leq 2 \lambda$ a.s. 
Further, to prove the second part of (i) we will use the representation of $M^{1}$ from the last line of (3.9). Notice that by [26, Theorem 7.12] and 23, Corollary 2.6.30] for each fixed $t \geq 0$

$$
\mathbb{E}\left\|M_{t}^{\tau}\right\| \leq \mathbb{E}\left\|M_{t}\right\|
$$

Moreover,

$$
\begin{aligned}
\mathbb{E}\left\|N_{t}\right\|=\mathbb{E}\left\|M_{t}^{\tau}-M_{t}^{\tau-}\right\| \leq \mathbb{E}\left\|M_{t}^{\tau}\right\|+\mathbb{E}\left(\left\|M_{t}^{\tau-}\right\| \mathbf{1}_{\tau<\infty}\right) \\
\quad \leq \mathbb{E}\left\|M_{t}^{\tau}\right\|+\mathbb{E}\left(\frac{\lambda}{2} \mathbf{1}_{\tau<\infty}\right) \leq 2 \mathbb{E}\left\|M_{t}^{\tau}\right\| \stackrel{(*)}{\leq} 2 \mathbb{E}\left\|M_{t}\right\|,
\end{aligned}
$$

where $\left\|M_{t}^{\tau-}\right\| \leq \frac{\lambda}{2} \leq\left\|M_{t}^{\tau}\right\|$ on $\{\tau<\infty\}$ by the definition of $\tau$, and (*) follows from [26, Theorem 7.12] and [23, Corollary 2.6.30]. Therefore by (3.2)

$$
\mathbb{E}\left\|V_{t}\right\| \leq \mathbb{E}\left\|N_{t}\right\| \leq 2 \mathbb{E}\left\|M_{t}\right\|
$$

as well. Finally, $\mathbb{E}\left\|M_{0}^{\tau-}\right\| \leq \mathbb{E}\left\|M_{0}\right\| \leq \mathbb{E}\left\|M_{t}\right\|$ by (3.7) and [23, Corollary 2.6.30]. Consequently, the second part of (i) holds by the estimates above and by the triangle inequality.

Step 2: $M^{2}$. First note that

$$
M^{2}=M-M^{\tau}+\left(M^{\tau-}+V\right)-\left(M^{\tau-}+V\right)^{\sigma-} .
$$

Let us check that $M^{2}$ is a martingale. $M-M^{\tau}$ is a martingale by [26, Theorem 7.12]. Furthermore,

$$
M^{\tau-}+V=M^{\tau}-(N-V)
$$

is a martingale as well due to [26, Theorem 7.12] and the fact that $V$ is a compensator of $N$. Finally, $\left(M^{\tau-}+V\right)^{\sigma-}$ is a martingale by Lemma 2.8 .

Let us now prove (ii). Notice that by (3.12)

$$
\mathbb{P}\left(\left(M^{2}\right)_{t}^{*}>0\right) \leq \mathbb{P}\left(\left(M-M^{\tau}\right)_{t}^{*}>0\right)+\mathbb{P}\left(\left(\left(M^{\tau-}+V\right)-\left(M^{\tau-}+V\right)^{\sigma-}\right)_{t}^{*}>0\right) .
$$

First estimate $\mathbb{P}\left(\left(M-M^{\tau}\right)_{t}^{*}>0\right)$ :

$$
\mathbb{P}\left(\left(M-M^{\tau}\right)_{t}^{*}>0\right) \leq \mathbb{P}(\tau \leq t) \leq \mathbb{P}\left(M_{t}^{*} \geq \frac{\lambda}{2}\right) \leq \frac{2 \mathbb{E}\left\|M_{t}\right\|}{\lambda},
$$

where the latter inequality holds by (2.1). Using the same machinery we get

$$
\begin{aligned}
\mathbb{P}\left(\left(\left(M^{\tau-}+V\right)-\left(M^{\tau-}+V\right)^{\sigma-}\right)_{t}^{*}>0\right) \leq & \mathbb{P}(\sigma \leq t) \\
& =\mathbb{P}\left(\left\|V_{t}\right\| \geq \lambda\right) \stackrel{(i)}{\leq} \frac{\mathbb{E}\left\|V_{t}\right\|}{\lambda} \stackrel{(i i)}{\leq} \frac{2 \mathbb{E}\left\|M_{t}\right\|}{\lambda},
\end{aligned}
$$

where (i) follows from the Chebyshev inequality, and (ii) follows from (3.11). This terminates the proof of (ii).

Step 3: $M^{3}$. Recall that

$$
M^{3}=M_{0}^{\tau-}+N-V .
$$

Therefore by the triangle inequality a.s. for each $t \geq 0$

$$
\begin{aligned}
\mathbb{E}\left(\operatorname{Var} M^{3}\right)_{t} & \leq \mathbb{E}\left\|M_{0}^{\tau-}\right\|+\mathbb{E}(\operatorname{Var} N)_{t}+\mathbb{E}(\operatorname{Var} V)_{t} \\
& \leq \mathbb{E}\left\|M_{t}\right\|+2 \mathbb{E}\left\|N_{t}\right\| \leq 5 \mathbb{E}\left\|M_{t}\right\|,
\end{aligned}
$$

where the latter inequality holds by (3.11), while the rest follows from (3.1) and the fact that $\mathbb{E}\left\|M_{0}^{\tau-}\right\| \leq \mathbb{E}\left\|M_{0}\right\| \leq \mathbb{E}\left\|M_{t}\right\|$. 
Remark 3.5. Let $p \in(1, \infty), M$ be an $L^{p}$-martingale, $\lambda>0, M=M^{1}+M^{2}+M^{3}$ be Gundy's decomposition (see the theorem above). Then $M^{1}$ is an $L^{p}$ martingale since $\left\|M_{t}^{1}\right\|_{L^{\infty}(\Omega ; X)} \leq 2 \lambda$ for all $t \geq 0 ; M^{3}$ is a local $L^{p}$-martingale since $M^{3}=$ $M_{0}^{\tau-}+N-V$, where both $M_{0}^{\tau-}$ and $N_{\infty}=\Delta M_{\tau}$ are $L^{p}$-integrable (the latter is $L^{p}$-integrable by the argument similar to (3.8)), and $V$ is locally $L^{p}$-integrable by Lemma 3.4 finally, $M^{2}$ is a local $L^{p}$-martingale since $M^{2}=M-M^{1}-M^{3}$. Therefore all the martingales in Gundy's decomposition are locally $L^{p}$-integrable given $M$ is an $L^{p}$-martingale.

\section{The CANonical DeCOMPosition of LOCAL MARTingales}

The current section is devoted to the proof of the fact that the canonical decomposition (as well as the Meyer-Yoeurp and the Yoeurp decompositions) of any $X$-valued local martingale exists if and only if $X$ has the UMD Banach property. Recall that the Meyer-Yoeurp decomposition split a local martingale $M$ into a sum $M=M^{c}+M^{d}$ of a continuous local martingale $M^{c}$ and a purely discontinuous local martingale $M^{d}$, while the Yoeurp decomposition split a purely discontinuous local martingale $M^{d}$ into a sum $M^{d}=M^{q}+M^{a}$ of a quasi-left continuous local martingale $M^{q}$ and a local martingale $M^{a}$ with accessible jumps.

First we give all the basic definitions properly, and thereafter we provide the reader with the proof of the main statement, Theorem 4.8 .

4.1. Basic definitions and decompositions of $L^{p}$-martingales. Let $X$ be a Banach space. Recall that a purely discontinuous local martingale have been defined in Subsection 2.3

Definition 4.1. A local martingale $M: \mathbb{R}_{+} \times \Omega \rightarrow X$ is called to have the MeyerYoeurp decomposition if there exist local martingales $M^{c}, M^{d}: \mathbb{R}_{+} \times \Omega \rightarrow X$ such that $M^{c}$ is continuous, $M^{d}$ is purely discontinuous, $M_{0}^{c}=0$, and $M=M^{c}+M^{d}$.

Remark 4.2. Recall that by [51] if $M=M^{c}+M^{d}$ is the Meyer-Yoeurp decomposition, then $\left\langle M^{c}, x^{*}\right\rangle$ is continuous and $\left\langle M^{d}, x^{*}\right\rangle$ is purely discontinuous for any $x^{*} \in X^{*}$; therefore this decomposition is unique by the uniqueness of the MeyerYoeurp decomposition of a real-valued local martingale (see [26, Theorem 26.14] and 51] for details).

Let $M: \mathbb{R}_{+} \times \Omega \rightarrow X$ be a local martingale. Then $M$ is called quasi-left continuous if $\Delta M_{\tau}=0$ a.s. for any predictable stopping time $\tau$, and $M$ is called with accessible jumps if $\Delta M_{\tau}=0$ a.s. for any totally inaccessible stopping time $\tau$ (see Subsection 2.5 for the definition of a predictable and a totally inaccessible stopping times).

Definition 4.3. A purely discontinuous local martingale $M^{d}: \mathbb{R}_{+} \times \Omega \rightarrow X$ is called to have the Yoeurp decomposition if there exist purely discontinuous local martingales $M^{q}, M^{a}: \mathbb{R}_{+} \times \Omega \rightarrow X$ such that $M^{q}$ is quasi-left continuous, $M^{a}$ has accessible jumps, $M_{0}^{q}=0$, and $M^{d}=M^{q}+M^{a}$.

Remark 4.4. Analogously to Remark 4.2 it follows from [26, Corollary 26.16] that the Yoeurp decomposition is unique.

Composing Definition 4.1 and 4.3 we get the canonical decomposition. 
Definition 4.5. A local martingale $M: \mathbb{R}_{+} \times \Omega \rightarrow X$ is called to have the canonical decomposition if there exist local martingales $M^{c}, M^{q}, M^{a}: \mathbb{R}_{+} \times \Omega \rightarrow X$ such that $M^{c}$ is continuous, $M^{q}$ and $M^{a}$ are purely discontinuous, $M^{q}$ is quasi-left continuous, $M^{a}$ has accessible jumps, $M_{0}^{c}=M_{0}^{q}=0$, and $M=M^{c}+M^{q}+M^{a}$.

Remark 4.6. Notice that if $M=M^{c}+M^{q}+M^{a}$ is the canonical decomposition, then $\Delta M_{\tau}^{q}=\Delta M_{\tau}$ for any totally inaccessible stopping time $\tau$ since in this case $\Delta M_{\tau}^{c}=\Delta M_{\tau}^{a}=0$ by the definition of a continuous local martingale and a local martingale with accessible jumps. Analogously, $\Delta M_{\tau}^{a}=\Delta M_{\tau}$ for any predictable stopping time $\tau$.

The reader can find further details on the martingale decomposition discussed above in [15, 25, 26, 36, 51, 54].

Due to [51] the UMD property guarantees the canonical decomposition of any $X$-valued $L^{p}$-martingale with $p \in(1, \infty)$ and the following proposition holds:

Proposition 4.7. Let $X$ be a $U M D$ Banach space, $p \in(1, \infty)$. Then any $L^{p}$-martingale $M: \mathbb{R}_{+} \times \Omega \rightarrow X$ has the canonical decomposition $M=M^{c}+M^{q}+M^{a}$, and then for each $t \geq 0$ we have that

$$
\begin{aligned}
& \mathbb{E}\left\|M_{t}^{c}\right\|^{p} \leq \beta_{p, X}^{p} \mathbb{E}\left\|M_{t}\right\|^{p}, \\
& \mathbb{E}\left\|M_{t}^{q}\right\|^{p} \leq \beta_{p, X}^{p} \mathbb{E}\left\|M_{t}\right\|^{p}, \\
& \mathbb{E}\left\|M_{t}^{a}\right\|^{p} \leq \beta_{p, X}^{p} \mathbb{E}\left\|M_{t}\right\|^{p},
\end{aligned}
$$

where $\beta_{p, X}$ is the $U M D_{p}$ constant of $X$.

It is a natural question whether the canonical decomposition is possible and whether one can extend (4.1) in the case $p=1$. It turns out that the UMD property is necessary and sufficient for the canonical decomposition of a general local martingale, while instead of (4.1) one gets weak-type estimates:

Theorem 4.8 (Canonical decomposition of local martingales). Let $X$ be a Banach space. Then $X$ has the UMD property if and only if any local martingale $M$ : $\mathbb{R}_{+} \times \Omega \rightarrow X$ has the canonical decomposition $M=M^{c}+M^{q}+M^{a}$. If this is the case, then for any $\lambda>0$ and $t \geq 0$

$$
\begin{aligned}
& \lambda \mathbb{P}\left(\left(M^{c}\right)_{t}^{*}>\lambda\right) \lesssim_{X} \mathbb{E}\left\|M_{t}\right\|, \\
& \lambda \mathbb{P}\left(\left(M^{q}\right)_{t}^{*}>\lambda\right) \lesssim_{X} \mathbb{E}\left\|M_{t}\right\|, \\
& \lambda \mathbb{P}\left(\left(M^{a}\right)_{t}^{*}>\lambda\right) \lesssim_{X} \mathbb{E}\left\|M_{t}\right\| .
\end{aligned}
$$

For the proof of the main theorem we will need a considerable amount of machinery, which will be provided in Subsection 4.24.4.

4.2. Weak differential subordination martingale transforms. The current subsection is devoted to the proof of the fact that boundedness of a continuoustime martingale transform from a certain specific class acting on $L^{p}$-martingales implies the corresponding weak $L^{1}$-estimates. Such type of assertions for special discrete martingale transforms was first obtained by Burkholder in [4]. Later the Burkholder's original statement was widely generalized in different directions (see [6, 11, 17, 22, 23, 33]), even though the martingale transforms were remaining acting on discrete martingales. The propose of the current section is to provide new results for martingale transforms of the same spirit by considering continuoustime martingales. This will allow us to consider linear operators that map a local 
martingale to the continuous part of the canonical decomposition, or the part of the canonical decomposition which is purely discontinuous with accessible jumps, so weak $L^{1}$-estimates (4.2) will follow from $L^{p}$-estimates (4.1) and Theorem 4.9

Before proving the main statement (Theorem 4.9) we need to provide the reader with basic definitions. Let $M: \mathbb{R}_{+} \times \Omega \rightarrow \mathbb{R}$ be a local martingale. We define a quadratic variation of $M$ in the following way:

$$
[M]_{t}:=\mathbb{P}-\lim _{\operatorname{mesh} \rightarrow 0} \sum_{n=1}^{N}\left|M\left(t_{n}\right)-M\left(t_{n-1}\right)\right|^{2},
$$

where the limit in probability is taken over partitions $0=t_{0}<\ldots<t_{N}=t$. The reader can find more about a quadratic variation in 25, 26, 35, 45].

Let $M, N: \mathbb{R}_{+} \times \Omega \rightarrow \mathbb{R}$ be local martingales. Then $N$ is called to be differentially subordinated to $M$ (or $N \ll M$ ) if $\left|N_{0}\right| \leq\left|M_{0}\right|$ a.s. and $[N]_{t}-[N]_{s} \leq[M]_{t}-[M]_{s}$ a.s. for each $0 \leq s \leq t<\infty$. We recommend the reader [1, 2, 7, [23, 40, 50] for further acquaintance with differential subordination.

Let $X$ be a Banach space, $M, N: \mathbb{R}_{+} \times \Omega \rightarrow X$ be local martingales. Then $N$ is called to be weakly differentially subordinated to $M$ (or $N \stackrel{w}{\ll} M$ ) if $\left\langle N, x^{*}\right\rangle$ is differentially subordinated to $\left\langle M, x^{*}\right\rangle$ for each $x^{*} \in X^{*}$. The reader can find more details on weak differential subordination in [41, 51, 52, 53].

The following theorem will be an important tool to show Theorem 4.8 and it is connected with [23, Proposition 3.5.4]. Recall that $\mathcal{M}_{X}^{p}$ is a space of all $L^{p}$-integrable $X$-valued martingales, and $\mathcal{M}_{X}^{p \text { loc }}$ is a space of all locally $L^{p}$-integrable $X$-valued martingales (see Subsection 2.2).

Theorem 4.9. Let $X$ be a Banach space, $p \in(1, \infty), T: \mathcal{M}_{X}^{p, \text { loc }} \rightarrow \mathcal{M}_{X}^{p \text {,loc }}$ be a linear operator such that $T M \stackrel{w}{\ll} M$ and

$$
M_{\infty}^{*}=0 \Longrightarrow(T M)_{\infty}^{*}=0 \quad \text { a.s. }
$$

for each $M \in \mathcal{M}_{X}^{p}$. Assume that $T \in \mathcal{L}\left(\mathcal{M}_{X}^{p}\right)$. Then for any $M \in \mathcal{M}_{X}^{p}$

$$
\lambda \mathbb{P}\left(\left\|(T M)_{\infty}^{*}\right\|>\lambda\right) \leq C_{p, T, X} \mathbb{E}\left\|M_{\infty}\right\|, \quad \lambda>0,
$$

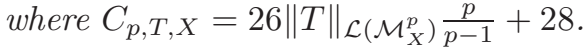

Remark 4.10. Notice that if $X$ is a UMD Banach space, then $T$ is automatically bounded on $\mathcal{M}_{X}^{p}$ and $\|T\|_{\mathcal{L}\left(\mathcal{M}_{X}^{p}\right)} \leq \beta_{p, X}^{2}\left(\beta_{p, X}+1\right)$ by [1.3) and [1] since $T M \stackrel{w}{\ll} M$ for any $M \in \mathcal{M}_{X}^{p}$.

For the proof we will need several lemmas.

Lemma 4.11. Let $X$ be a Banach space, $M: \mathbb{R}_{+} \times \Omega \rightarrow X$ be a purely discontinuous martingale with $M_{0}=0$ a.s. Let $\mu^{M}$ be the corresponding random measure defined as in (3.3). Assume that

$$
\mathbb{E} \sum_{s \geq 0}\left\|\Delta M_{s}\right\|=\mathbb{E} \int_{\mathbb{R}_{+} \times X}\|x\| \mathrm{d} \mu^{M}<\infty .
$$

Then $M_{t}=\int_{[0, t] \times X} x \mathrm{~d} \bar{\mu}^{M}$ for each $t \geq 0$ a.s. 
Proof. By (4.6) there exists $N: \mathbb{R}_{+} \times \Omega \rightarrow X$ such that $N_{t}=\sum_{0 \leq s \leq t} \Delta M_{s}$ for each $t \geq 0$. Let $V=N-M$. Then both $t \mapsto N_{t}-V_{t}=M_{t}, t \geq 0$, and

$t \mapsto N_{t}-\int_{[0, t] \times X} x \mathrm{~d} \nu^{M}=\int_{[0, t] \times X} x \mathrm{~d} \mu^{M}-\int_{[0, t] \times X} x \mathrm{~d} \nu^{M}=\int_{[0, t] \times X} x \mathrm{~d} \bar{\mu}^{M}, \quad t \geq 0$, are martingales. Therefore

$$
t \mapsto V_{t}-\int_{[0, t] \times X} x \mathrm{~d} \nu^{M}=M_{t}-\int_{[0, t] \times X} x \mathrm{~d} \bar{\mu}^{M}, \quad t \geq 0,
$$

is a predictable martingale, which is purely discontinuous as a difference of two purely discontinuous martingales (see Lemma 2.6). On the other hand it is continuous by the predictability (see e.g. 29, Theorem 4] and [27, Corollary 2.1.42]). Hence by Lemma 2.5 this martingale equals zero since it starts at zero, so $M=$ $N-V=\int_{[0, \cdot] \times X} x \mathrm{~d} \bar{\mu}^{M}$.

Lemma 4.12. Let $X$ be a Banach space, $M, N: \mathbb{R}_{+} \times \Omega \rightarrow X$ be purely discontinuous martingales such that $N \stackrel{w}{\ll} M$. Then $\mathbb{E}(\operatorname{Var} N)_{t} \leq 2 \mathbb{E}(\operatorname{Var} M)_{t}$ for each $t \geq 0$.

Proof. Without loss of generality $\mathbb{E}(\operatorname{Var} M)_{\infty}<\infty$. Notice that since $N \stackrel{w}{\ll} M$, for a.e. $(t, \omega) \in \mathbb{R}_{+} \times \Omega$ there exists $a(t, \omega) \in[-1,1]$ such that $\Delta N_{t}(\omega)=a(t, \omega) \Delta M_{t}(\omega)$ (see [53]). Therefore a.s. for each $t \geq 0$

$$
\begin{aligned}
\int_{[0, t] \times X}\|x\| \mathrm{d} \mu^{N}(x, s) & =\sum_{0 \leq s \leq t}\left\|\Delta N_{s}\right\|=\sum_{0 \leq s \leq t}|a(s, \cdot)|\left\|\Delta M_{s}\right\| \\
& \leq \sum_{0 \leq s \leq t}\left\|\Delta M_{s}\right\| \leq(\operatorname{Var} M)_{t} .
\end{aligned}
$$

So by Lemma $4.11 N=\int_{[0, \cdot] \times X} x \mathrm{~d} \bar{\mu}^{N}$, hence

$$
\begin{aligned}
(\operatorname{Var} N)_{t} & =\left(\operatorname{Var} \int_{[0, \cdot] \times X} x \mathrm{~d} \bar{\mu}^{N}(x, s)\right)_{t} \\
& =\left(\operatorname{Var}\left(\int_{[0, \cdot] \times X} x \mathrm{~d} \mu^{N}(x, s)-\int_{[0, \cdot] \times X} x \mathrm{~d} \nu^{N}(x, s)\right)\right)_{t} \\
& \leq\left(\operatorname{Var} \int_{[0, \cdot] \times X} x \mathrm{~d} \mu^{N}(x, s)\right)_{t}+\left(\operatorname{Var} \int_{[0, \cdot] \times X} x \mathrm{~d} \nu^{N}(x, s)\right)_{t} \\
& \leq \int_{[0, t] \times X}\|x\| \mathrm{d} \mu^{N}(x, s)+\int_{[0, t] \times X}\|x\| \mathrm{d} \nu^{N}(x, s) \\
& =2 \int_{[0, t] \times X}\|x\| \mathrm{d} \mu^{N}(x, s) \stackrel{(*)}{\leq} 2(\operatorname{Var} M)_{t},
\end{aligned}
$$

where $(*)$ holds by (4.7).

Proof of Theorem 4.9. The proof has the same structure as the proof of 23, Proposition 3.5.16]. Fix $M \in \mathcal{M}_{X}^{p}$ and $\lambda>0$. Let $K:=\|T\|_{\mathcal{L}\left(\mathcal{M}_{X}^{p}\right)}, M=M^{1}+M^{2}+M^{3}$ be Gundy's decomposition of $M$ from Theorem 3.1 at the level $\alpha \lambda$ for some $\alpha>0$ which we will fix later. Notice that all $M^{1}, M^{2}$ and $M^{3}$ are local $L^{p}$-martingales by Remark 3.5. Then

$$
\mathbb{P}\left(\left\|(T M)_{\infty}^{*}\right\|>\lambda\right)
$$




$$
\leq \mathbb{P}\left(\left\|\left(T M^{1}\right)_{\infty}^{*}\right\|>\frac{\lambda}{2}\right)+\mathbb{P}\left(\left\|\left(T M^{2}\right)_{\infty}^{*}\right\|>0\right)+\mathbb{P}\left(\left\|\left(T M^{3}\right)_{\infty}^{*}\right\|>\frac{\lambda}{2}\right)
$$

Let us estimate each of these three terms separately. First,

$$
\begin{aligned}
\mathbb{P}\left(\left\|\left(T M^{1}\right)_{\infty}^{*}\right\|>\frac{\lambda}{2}\right) & \stackrel{(i)}{\leq}\left(\frac{2}{\lambda}\right)^{p} \mathbb{E}\left\|\left(T M^{1}\right)_{\infty}^{*}\right\|^{p} \stackrel{(*)}{\leq}\left(\frac{2}{\lambda} \frac{p}{p-1}\right)^{p} \mathbb{E}\left\|\left(T M^{1}\right)_{\infty}\right\|^{p} \\
& \stackrel{(i i)}{\leq}\left(\frac{2 K}{\lambda} \frac{p}{p-1}\right)^{p} \mathbb{E}\left\|M_{\infty}^{1}\right\|^{p} \leq\left(\frac{2 K}{\lambda} \frac{p}{p-1}\right)^{p}\left\|M_{\infty}^{1}\right\|_{\infty}^{p-1} \mathbb{E}\left\|M_{\infty}^{1}\right\| \\
& \stackrel{(i i i)}{\leq}\left(\frac{2 K}{\lambda} \frac{p}{p-1}\right)^{p}(2 \alpha \lambda)^{p-1} 5 \mathbb{E}\left\|M_{\infty}\right\|=\frac{5\left(4 \alpha K \frac{p}{p-1}\right)^{p}}{2 \alpha \lambda} \mathbb{E}\left\|M_{\infty}\right\|,
\end{aligned}
$$

where $(i)$ follows from (2.1) $(*)$ follows from Doob's maximal inequality 28 , Theorem 1.3.8(iv)], (ii) holds by the definition of $K$, and (iii) follows from Gundy's decomposition.

Now turn to $M^{2}$. By (4.4)

$$
\mathbb{P}\left(\left(T M^{2}\right)_{\infty}^{*}>0\right) \leq \mathbb{P}\left(\left(M^{2}\right)_{\infty}^{*}>0\right) \leq \frac{4}{\alpha \lambda} \mathbb{E}\left\|M_{\infty}\right\| .
$$

Finally, by Lemma 4.12 and the fact that $T M^{3} \stackrel{w}{\ll} M^{3}$ we have that

$$
\mathbb{E}\left(\operatorname{Var} T M^{3}\right)_{\infty} \leq 2 \mathbb{E}\left(\operatorname{Var} M^{3}\right)_{\infty},
$$

hence

$$
\begin{aligned}
\mathbb{P}\left(\left\|\left(T M^{3}\right)_{\infty}^{*}\right\|>\frac{\lambda}{2}\right) & \stackrel{(i)}{\leq} \frac{2}{\lambda} \mathbb{E}\left\|\left(T M^{3}\right)_{\infty}^{*}\right\| \leq \frac{2}{\lambda} \mathbb{E}\left(\operatorname{Var} T M^{3}\right)_{\infty} \\
& \stackrel{(i i)}{\leq} \frac{4}{\lambda} \mathbb{E}\left(\operatorname{Var} M^{3}\right)_{\infty} \stackrel{(*)}{\leq} \frac{28}{\lambda} \mathbb{E}\left\|M_{\infty}\right\|,
\end{aligned}
$$

where $(i)$ follows from (2.1), (ii) holds by (4.9), and $(*)$ holds by Theorem 3.1(iii). Therefore by (4.8)

$$
\begin{aligned}
\lambda \mathbb{P}\left(\left\|(T M)_{\infty}^{*}\right\|>\lambda\right) & \leq \lambda\left(\frac{5\left(4 \alpha K \frac{p}{p-1}\right)^{p}}{2 \alpha \lambda}+\frac{4}{\alpha \lambda}+\frac{28}{\lambda}\right) \mathbb{E}\left\|M_{\infty}\right\| \\
& =\left(\frac{5\left(4 \alpha K \frac{p}{p-1}\right)^{p}}{2 \alpha}+\frac{4}{\alpha}+28\right) \mathbb{E}\left\|M_{\infty}\right\|,
\end{aligned}
$$

and by choosing $\alpha=\frac{p-1}{4 K p}$ we get

$$
\begin{aligned}
\lambda \mathbb{P}\left(\left\|(T M)_{\infty}^{*}\right\|>\lambda\right) & \leq\left(10 K \frac{p}{p-1}+16 K \frac{p}{p-1}+28\right) \mathbb{E}\left\|M_{\infty}\right\| \\
& =\left(26 K \frac{p}{p-1}+28\right) \mathbb{E}\left\|M_{\infty}\right\|,
\end{aligned}
$$

which is exactly (4.5).

The following proposition shows that the operator $T$ from Theorem 4.9 has a special structure given the filtration $\mathbb{F}=\left(\mathcal{F}_{t}\right)_{t \geq 0}$ is generated by $\left(\mathcal{F}_{n}\right)_{n \geq 0}$ : such martingale transforms are the same as those considered in 23, Proposition 3.5.4] and [6].

Proposition 4.13. Let $X$ be a separable Banach space. Let the filtration $\mathbb{F}=$ $\left(\mathcal{F}_{t}\right)_{t \geq 0}$ be of the following form: $\mathcal{F}_{t}=\mathcal{F}_{\lfloor t\rfloor}$ for each $t \geq 0, T$ be as in Theorem 4.9. Then there exists an $\left(\mathcal{F}_{n}\right)_{n \geq 0}$-predictable sequence $\left(a_{n}\right)_{n \geq 0}$ with values in $[-1,1]$ such that $\Delta(T M)_{n}=a_{n} \Delta M_{n}$ a.s. for each $n \geq 0$ for any $M \in \mathcal{M}_{X}^{p}$. 
Proof. Let $\mathbb{G}=\left(\mathcal{G}_{n}\right)_{n \geq 0}:=\left(\mathcal{F}_{n}\right)_{n \geq 0}$ be a discrete filtration. Due to the construction of $\mathbb{F}$ and the fact that $\mathbb{G}$ is discrete we have that any $\mathbb{F}$-martingale $M$ is in fact discrete (i.e. $M_{t}=M_{\lfloor t\rfloor}$ a.s. for each $t \geq 0$ ), hence any martingale has accessible jumps, so by Lemma 4.20 it is sufficient to use the fact that $T M \stackrel{w}{\ll} M$ for any $M \in \mathcal{M}_{X}^{p}$ in order to apply Theorem 4.9, Let us show that there exists a $\mathbb{G}$-adapted $[-1,1]$-valued sequence $\left(a_{n}\right)_{n \geq 1}$ such that $\Delta(T M)_{n}=a_{n} \Delta M_{n}$ a.s. for each $n \geq 0$. Since $X$ is separable, $L^{p}(\Omega ; X)$ is separable by 23 , Proposition 1.2.29]. Let $\left(\xi^{m}\right)_{m \geq 1}$ be a dense subset of $L^{p}(\Omega ; X)$. For each $m \geq 1$ we construct a martingale $M^{m}$ in the following way: $M_{t}^{m}:=\mathbb{E}\left(\xi^{m} \mid \mathcal{F}_{t}\right), t \geq 0$. Then we have that $\left((T M)_{n}^{m}\right)_{n \geq 0} \stackrel{w}{\ll}\left(M_{n}^{m}\right)_{n \geq 0}$ for each $m \geq 1$, so by [53] for each $m \geq 1$ there exists a $\mathbb{G}$-adapted $[-1,1]$-valued sequence $\left(a_{n}^{m}\right)_{n \geq 0}$ such that $\Delta\left(T M^{m}\right)_{n}=a_{n}^{m} \Delta M_{n}^{m}$ for each $n \geq 0$. Let us show that for each $m_{1} \neq m_{2}$ and $n \geq 0$ we have that

$$
a_{n}^{m_{1}}=a_{n}^{m_{2}} \text { a.s. on } A_{n}^{m_{1}, m_{2}},
$$

where $A_{n}^{m_{1}, m_{2}}:=\left\{\Delta M_{n}^{m_{1}} \neq 0\right\} \cap\left\{\Delta M_{n}^{m_{2}} \neq 0\right\}$. Let $\left(\left(c_{1}^{k}, c_{2}^{k}\right)\right)_{k>1}$ be a dense subset of $\mathbb{R}^{2}$ such that for each $k \geq 1$

$$
c_{1}^{k} \Delta M_{n}^{m_{1}}+c_{2}^{k} \Delta M_{n}^{m_{2}} \neq 0 \text { a.s. on } A_{n}^{m_{1}, m_{2}} .
$$

Then $T\left(c_{1}^{k} M^{m_{1}}+c_{2}^{k} M^{m_{2}}\right) \stackrel{w}{\ll} c_{1}^{k} M^{m_{1}}+c_{2}^{k} M^{m_{2}}$ for each $k \geq 1$, and hence by the linearity of $T$ we have that for each $k \geq 1$ a.s. $c_{1}^{k} a_{n}^{m_{1}} \Delta M_{n}^{m_{1}}+c_{2}^{k} a_{n}^{m_{2}} \Delta M_{n}^{m_{2}}$ and $c_{1}^{k} \Delta M_{n}^{m_{1}}+c_{2}^{k} \Delta M_{n}^{m_{2}}$ are collinear vectors in $X$, and

$$
\left|\frac{c_{1}^{k} a_{n}^{m_{1}} \Delta M_{n}^{m_{1}}+c_{2}^{k} a_{n}^{m_{2}} \Delta M_{n}^{m_{2}}}{c_{1}^{k} \Delta M_{n}^{m_{1}}+c_{2}^{k} \Delta M_{n}^{m_{2}}}\right| \leq 1 \text { a.s. on } A_{n}^{m_{1}, m_{2}}
$$

by the weak differential subordination. Therefore we can redefine $A_{n}^{m_{1}, m_{2}}$ up to a negligible set in the following way:

$$
\begin{aligned}
A_{n}^{m_{1}, m_{2}}:=A_{n}^{m_{1}, m_{2}} & \bigcap_{k \geq 1}\left\{c_{1}^{k} \Delta M_{n}^{m_{1}}+c_{2}^{k} \Delta M_{n}^{m_{2}} \neq 0\right\} \\
& \bigcap_{k \geq 1}\left\{\left|\frac{c_{1}^{k} a_{n}^{m_{1}} \Delta M_{n}^{m_{1}}+c_{2}^{k} a_{n}^{m_{2}} \Delta M_{n}^{m_{2}}}{c_{1}^{k} \Delta M_{n}^{m_{1}}+c_{2}^{k} \Delta M_{n}^{m_{2}}}\right| \leq 1\right\} .
\end{aligned}
$$

Let us now fix any $\omega \in A_{n}^{m_{1}, m_{2}}$ and $\varepsilon>0$. Let $x^{*} \in X^{*}$ be such that $\left\langle\Delta M_{n}^{m_{1}}(\omega), x^{*}\right\rangle \neq 0$ and $\left\langle\Delta M_{n}^{m_{2}}(\omega), x^{*}\right\rangle \neq 0$ (such $x^{*}$ exists by the Hahn-Banach theorem and the definition of $\left.A_{n}^{m_{1}, m_{2}}\right)$. Then we can find $k \geq 1$ such that

$$
0<\frac{\left\langle c_{1}^{k} \Delta M_{n}^{m_{1}}(\omega)+c_{2}^{k} \Delta M_{n}^{m_{2}}(\omega), x^{*}\right\rangle}{\left|c_{1}^{k}\right|+\left|c_{2}^{k}\right|}<\varepsilon
$$

since $\left(\left(c_{1}^{k}, c_{2}^{k}\right)\right)_{k>1}$ is dense in $\mathbb{R}^{2}$ (i.e. $k \geq 0$ such that $\left(c_{1}^{k}, c_{2}^{k}\right)$ is almost orthogonal to $\left.\left(\left\langle\Delta M_{n}^{m_{1}}(\omega) x^{*}\right\rangle,\left\langle\Delta M_{n}^{m_{2}}(\omega), x^{*}\right\rangle\right)\right)$. But on the other hand (we will omit $\omega$ for the convenience of the reader)

$$
\begin{aligned}
1 & \geq\left|\frac{c_{1}^{k} a_{n}^{m_{1}} \Delta M_{n}^{m_{1}}+c_{2}^{k} a_{n}^{m_{2}} \Delta M_{n}^{m_{2}}}{c_{1}^{k} \Delta M_{n}^{m_{1}}+c_{2}^{k} \Delta M_{n}^{m_{2}}}\right|=\frac{\left|\left\langle c_{1}^{k} a_{n}^{m_{1}} \Delta M_{n}^{m_{1}}+c_{2}^{k} a_{n}^{m_{2}} \Delta M_{n}^{m_{2}}, x^{*}\right\rangle\right|}{\left\langle c_{1}^{k} \Delta M_{n}^{m_{1}}+c_{2}^{k} \Delta M_{n}^{m_{2}}, x^{*}\right\rangle} \\
& =\frac{\left|\left\langle c_{2}^{k}\left(a_{n}^{m_{2}}-a_{n}^{m_{1}}\right) \Delta M_{n}^{m_{2}}, x^{*}\right\rangle\right|-\left|\left\langle c_{1}^{k} a_{n}^{m_{1}} \Delta M_{n}^{m_{1}}+c_{2}^{k} a_{n}^{m_{1}} \Delta M_{n}^{m_{2}}, x^{*}\right\rangle\right|}{\left\langle c_{1}^{k} \Delta M_{n}^{m_{1}}+c_{2}^{k} \Delta M_{n}^{m_{2}}, x^{*}\right\rangle} \\
& \stackrel{(*)}{\geq}\left|a_{n}^{m_{2}}-a_{n}^{m_{1}}\right|\left|\left\langle\Delta M_{n}^{m_{2}}, x^{*}\right\rangle\right| \frac{1}{\varepsilon}-1,
\end{aligned}
$$


where (*) holds by the triangle inequality, 4.11), and the fact that $\left|a_{n}^{m_{1}}\right| \leq 1$. Since $\varepsilon$ was arbitrary, (4.12) holds true if and only if $a_{n}^{m_{2}}(\omega)-a_{n}^{m_{1}}(\omega)=0$. Now since $\omega \in A_{n}^{m_{1}, m_{2}}$ was arbitrary, $a_{n}^{m_{1}}=a_{n}^{m_{2}}$ on $A_{n}^{m_{1}, m_{2}}$.

Now we define for each $n \geq 0$ and $m \geq 1$ :

$$
\begin{aligned}
B_{n}^{1} & =\left\{\Delta M_{n}^{1} \neq 0\right\}, \\
B_{n}^{m} & =\left\{\Delta M_{n}^{m} \neq 0\right\} \backslash B_{n}^{m-1}, \quad m \geq 2, \\
B_{n}^{0} & =\Omega \backslash \bigcup_{m \geq 1} B_{n}^{m},
\end{aligned}
$$

and define $a_{n}$ in the following way:

$$
\begin{aligned}
& a_{n}(\omega):=a_{n}^{m}, \quad \omega \in B_{n}^{m}, m \geq 1, \\
& a_{n}(\omega):=0, \quad \omega \in B_{0}^{m} .
\end{aligned}
$$

Then by (4.10) $a_{n}=a_{n}^{m}$ a.s. on $\left\{\Delta M_{n}^{m} \neq 0\right\}$ for all $m \geq 1$. Therefore $\Delta\left(T M^{m}\right)_{n}=$ $a_{n} \Delta M_{n}^{m}$ a.s. for all $m \geq 1$. Now let $M$ be a general $L^{p}$-martingale. Let $\left(M^{m_{k}}\right)_{k \geq 1}$ be a sequence which converges to $M$ in $\mathcal{M}_{X}^{p}$. Fix $n \geq 0$. Then by [23, Corollary 2.6.30] $\Delta M_{n}^{m_{k}}$ converges to $\Delta M_{n}$ in $L^{p}(\Omega ; X)$ as $k \rightarrow \infty$, so by boundedness of $a_{n}$ we have that $a_{n} \Delta M_{n}^{m_{k}} \rightarrow a_{n} \Delta M_{n}$ in $L^{p}(\Omega ; X)$. On the other hand by boundedness of $T$ and by [23, Corollary 2.6.30]

$$
\lim _{k \rightarrow \infty} a_{n} \Delta M_{m}^{n_{k}}=\lim _{k \rightarrow \infty} \Delta\left(T M_{n}^{m_{k}}\right)_{n}=\Delta(T M)_{n},
$$

where the limit is taken in $L^{p}(\Omega ; X)$. Hence $\Delta(T M)_{n}=a_{n} \Delta M_{n}$ a.s.

It follows from (4.13) and [53] that $\left(a_{n}\right)_{n \geq 0}$ is $\mathbb{G}$-adapted and bounded by 1 . Now let us show that $\left(a_{n}\right)_{n \geq 0}$ is $\mathbb{G}$-predictable. Assume the opposite. Then there

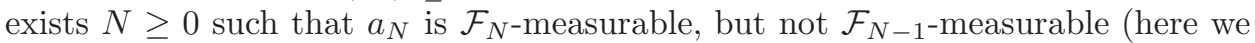
set $\mathcal{F}_{-1}$ to be the $\sigma$-algebra generated by all negligible sets). Fix $x \in X \backslash\{0\}$. Then we can construct the following $L^{p}$-martingale $M: \mathbb{R}_{+} \times \Omega \rightarrow X: \Delta M_{n}=0$ if $n \neq N$ and $\Delta M_{N}=\left(a_{N}-\mathbb{E}\left(a_{N} \mid \mathcal{F}_{N-1}\right)\right) x$. This is an $L^{p}$-martingale since by the triangle inequality and $[3$, Theorem 34.2]

$$
\begin{aligned}
\left\|a_{N}-\mathbb{E}\left(a_{N} \mid \mathcal{F}_{N-1}\right)\right\|_{\infty} & \leq\left\|a_{N}\right\|_{\infty}+\left\|\mathbb{E}\left(a_{N} \mid \mathcal{F}_{N-1}\right)\right\|_{\infty} \leq 1+\left\|\mathbb{E}\left(\mid a_{N} \| \mathcal{F}_{N-1}\right)\right\|_{\infty} \\
& \leq 1+\left\|\mathbb{E}\left(1 \mid \mathcal{F}_{N-1}\right)\right\|_{\infty} \leq 2 .
\end{aligned}
$$

Then we have that $\Delta(T M)_{N}=a_{N}\left(a_{N}-\mathbb{E}\left(a_{N} \mid \mathcal{F}_{N-1}\right)\right) x$, and since $T M$ is a martingale,

$$
\begin{aligned}
0=\mathbb{E}\left(\Delta(T M)_{N} \mid \mathcal{F}_{N-1}\right) & =\mathbb{E}\left(a_{N}\left(a_{N}-\mathbb{E}\left(a_{N} \mid \mathcal{F}_{N-1}\right)\right) x \mid \mathcal{F}_{N-1}\right) \\
& =x \mathbb{E}\left(a_{N}^{2}-a_{N} \mathbb{E}\left(a_{N} \mid \mathcal{F}_{N-1}\right) \mid \mathcal{F}_{N-1}\right) \\
& =x\left(\mathbb{E}\left(a_{N}^{2} \mid \mathcal{F}_{N-1}\right)-\left(\mathbb{E}\left(a_{N} \mid \mathcal{F}_{N-1}\right)\right)^{2}\right) \\
& =x \mathbb{E}\left(\left(a_{N}-\mathbb{E}\left(a_{N} \mid \mathcal{F}_{N-1}\right)\right)^{2} \mid \mathcal{F}_{N-1}\right),
\end{aligned}
$$

so since $x \neq 0$ and the fact that $\left(a_{N}-\mathbb{E}\left(a_{N} \mid \mathcal{F}_{N-1}\right)\right)^{2}$ is nonnegative we get that $a_{N}-\mathbb{E}\left(a_{N} \mid \mathcal{F}_{N-1}\right)=0$ a.s., hence $a_{N}$ is $\mathcal{F}_{N-1}$-measurable.

Remark 4.14. One can extend Proposition 4.13 to the case of a Banach space $X$ being over the scalar field $\mathbb{C}$. The point is that because of the structure of the filtration $\mathbb{F}$ any $\mathbb{F}$-martingale is purely discontinuous, so one can extend the definition of weak differential subordination in the way presented in [52]; namely, $N \stackrel{w}{\ll} M$ if 
$\left|\left\langle\Delta N_{t}, x^{*}\right\rangle\right| \leq\left|\left\langle\Delta M_{t}, x^{*}\right\rangle\right|$ a.s. for all $t \geq 0$ and $x^{*} \in X^{*}$. Then by applying the same proof one can show that the sequence $\left(a_{n}\right)_{n \geq 0}$ from Proposition 4.13 exists and is still $\left(\mathcal{F}_{n}\right)_{n \geq 0}$-predictable, but it takes values in the unit disk $\mathbb{D}:=\{\lambda \in \mathbb{C}:|\lambda| \leq 1\}$.

4.3. Sufficiency of the UMD property. Now we will consider two examples of an operator $T$ from Theorem 4.9, which will provide us with the Meyer-Yoeurp and the Yoeurp decompositions of any UMD space-valued local martingale.

Theorem 4.15 (Meyer-Yoeurp decomposition of local martingales). Let $X$ be a UMD Banach space, $M: \mathbb{R}_{+} \times \Omega \rightarrow X$ be a local martingale. Then there exist unique local martingales $M^{c}, M^{d}: \mathbb{R}_{+} \times \Omega \rightarrow X$ such that $M^{c}$ is continuous, $M^{d}$ is purely discontinuous, $M_{0}^{c}=0$, and $M=M^{c}+M^{d}$. Moreover, for any $\lambda>0$ and $t \geq 0$

$$
\begin{aligned}
& \lambda \mathbb{P}\left(\left(M^{c}\right)_{t}^{*}>\lambda\right) \lesssim X \mathbb{E}\left\|M_{t}\right\|, \\
& \lambda \mathbb{P}\left(\left(M^{d}\right)_{t}^{*}>\lambda\right) \lesssim X \mathbb{E}\left\|M_{t}\right\| .
\end{aligned}
$$

For the proof we will need the following lemma.

Lemma 4.16. Let $M: \mathbb{R}_{+} \times \Omega \rightarrow X$ be an $L^{1}$-martingale, $\left(M^{n}\right)_{n \geq 1}$ be a sequence of purely discontinuous $X$-valued $L^{1}$-martingales such that $M_{\infty}^{n} \rightarrow M_{\infty}$ in $L^{1}(\Omega ; X)$. Then $M$ is purely discontinuous.

Proof. Without loss of generality $M_{0}=0$ and $M_{0}^{n}=0$ a.s. for each $n \geq 1$. By Proposition 2.4 it is sufficient to check that $M N$ is a martingale for any bounded continuous real-valued martingale $N$ with $N_{0}=0$ a.s. Fix such $N$. Then due to Proposition 2.4 $M^{n} N$ is a martingale for each $n \geq 0$. Moreover, since $N_{t}$ is bounded for each $t \geq 0,\left(M^{n} N\right)_{t} \rightarrow(M N)_{t}$ in $L^{1}(\Omega ; X)$. Therefore by the boundedness of a conditional expectation operator (see [23, Corollary 2.6.30]) for each $0 \leq s \leq t$

$$
\begin{aligned}
\mathbb{E}\left((M N)_{t} \mid \mathcal{F}_{s}\right)=\mathbb{E}\left(\lim _{n \rightarrow \infty}\left(M^{n} N\right)_{t} \mid \mathcal{F}_{s}\right) & =\lim _{n \rightarrow \infty} \mathbb{E}\left(\left(M^{n} N\right)_{t} \mid \mathcal{F}_{s}\right) \\
& =\lim _{n \rightarrow \infty}\left(M^{n} N\right)_{s}=(M N)_{s} .
\end{aligned}
$$

Hence, $M N$ is a martingale. Since $N$ was arbitrary, $M$ is a purely discontinuous martingale.

Proof of Theorem 4.15. By a stopping time argument we can assume that $M$ is an $L^{1}$-martingale. Fix $p \in(1, \infty)$. Let $\left(M^{n}\right)_{n \geq 1}$ be a sequence of $X$-valued $L^{p_{-}}$ martingales such that $M_{\infty}^{n} \rightarrow M_{\infty}$ in $L^{1}(\Omega ; X)$. Without loss of generality assume that $\mathbb{E}\left\|M_{\infty}-M_{\infty}^{n}\right\|<\frac{1}{2^{n+1}}$ for each $n \geq 1$. Let $T \in \mathcal{L}\left(\mathcal{M}_{X}^{p}\right)$ be such that $T$ maps an $L^{p}$-martingale $N: \mathbb{R}_{+} \times \Omega \rightarrow X$ to its continuous part $N^{c}$ (such an operator exists and bounded by Proposition 4.7). For each $n \geq 1$ we denote $T M^{n}$ by $M^{n, c}$. Then we know that by Theorem 4.9 for each $m \geq n \geq 1$ and any $K>0$

$$
\mathbb{P}\left(\left(M^{n, c}-M^{m, c}\right)_{\infty}^{*}>K\right) \lesssim_{p, X} \frac{1}{K} \mathbb{E}\left\|M_{\infty}^{n, c}-M_{\infty}^{m, c}\right\| \leq \frac{1}{2^{n} K},
$$

hence $\left(M^{n, c}\right)_{n \geq 1}$ is a Cauchy sequence in the ucp topology by (2.2). Notice that all the $M^{n, c}$ s are continuous local martingales, which are complete in the ucp topology (see [49, pp. 7-8] and Lemma 2.3). Hence there exists a local martingale $M^{c}: \mathbb{R}_{+} \times \Omega \rightarrow X$ which is the limit of $\left(M^{n, c}\right)_{n \geq 1}$ in the ucp topology. Now it is sufficient to prove that $M_{0}^{c}=0$ and that $\left\langle M-M^{c}, x^{*}\right\rangle$ is a purely discontinuous local martingale for any $x^{*} \in X^{*}$ in order to show that $M^{c}$ is the desired continuous local martingale. Firstly, $M_{0}^{c}=\mathbb{P}-\lim _{n \rightarrow \infty} M_{0}^{n, c}=0$ since $M^{c}$ is the limit of $\left(M^{n, c}\right)_{n \geq 1}$ 
in the ucp topology and since $M_{0}^{n, c}=0$ a.s. for each $n \geq 1$. Secondly, since $M^{n, c} \rightarrow M^{c}$ in the ucp topology and $M^{n} \rightarrow M$ in $L^{1}(\Omega ; X),\left\langle M^{n}-M^{n, c}, x^{*}\right\rangle \rightarrow$ $\left\langle M-M^{c}, x^{*}\right\rangle$ in the ucp topology for each fixed $x^{*} \in X^{*}$. Without loss of generality set that $\mathbb{E}\left\|M_{\infty}\right\|, \mathbb{E}\left\|M_{\infty}^{n}\right\| \leq 1$ for each $n \geq 1$. Also by choosing a subsequence we can assume that $M^{c, n} \rightarrow M^{c}$ a.s. uniformly on compacts. Therefore by Lemma 2.3 the process $t \mapsto \sup _{0 \leq s \leq t} \sup _{n}\left\|M^{c, n}\right\|$ exists and continuous, and for each $m \geq 1$ we can define a stopping time $\tau_{m}$ in the following way

$$
\tau_{m}:=\inf \left\{t \geq 0: \sup _{0 \leq s \leq t} \sup _{n}\left\|M^{c, n}\right\| \geq m\right\} .
$$

Notice that a.s. $\tau_{m} \rightarrow \infty$ as $m \rightarrow \infty$. First show that $\left\langle\left(M-M^{c}\right)^{\tau_{m}}, x^{*}\right\rangle$ is purely discontinuous for each $m \geq 1$. Note that $\left(M^{c, n}\right)_{\infty}^{\tau_{m}} \rightarrow\left(M^{c}\right)_{\infty}^{\tau_{m}}$ and $\left(M^{n}\right)_{\infty}^{\tau_{m}} \rightarrow M_{\infty}^{\tau_{m}}$ in $L^{1}(\Omega ; X)$ as $n \rightarrow \infty$. Therefore

$$
\left\langle\left(M^{n}-M^{c, n}\right)^{\tau_{m}}, x^{*}\right\rangle \rightarrow\left\langle\left(M-M^{c}\right)^{\tau_{m}}, x^{*}\right\rangle
$$

in $L^{1}(\Omega)$, so by Lemma 4.16 $\left\langle\left(M-M^{c}\right)^{\tau_{m}}, x^{*}\right\rangle$ is purely discontinuous. Notice that by letting $m$ to infinity we get that $\left\langle M-M^{c}, x^{*}\right\rangle$ is a purely discontinuous local martingale for any $x^{*} \in X^{*}$, hence $M-M^{c}$ is a purely discontinuous local martingale.

The uniqueness of the decomposition follows from Remark 4.2, while (4.14) holds due to the limiting argument, (4.15), and the completeness of $L^{1, \infty}$-spaces provided by (1.1.11) and Theorem 1.4.11 in [18].

Let us turn to the Yoeurp decomposition.

Theorem 4.17 (Yoeurp decomposition of local martingales). Let $X$ be a UMD Banach space, $M^{d}: \mathbb{R}_{+} \times \Omega \rightarrow X$ be a purely discontinuous local martingale. Then there exist unique purely discontinuous local martingales $M^{q}, M^{a}: \mathbb{R}_{+} \times \Omega \rightarrow X$ such that $M^{q}$ is quasi-left continuous, $M^{a}$ has accessible jumps, $M_{0}^{q}=0$, and $M^{d}=M^{q}+M^{a}$. Moreover, for any $\lambda>0$ and $t \geq 0$

$$
\begin{aligned}
& \lambda \mathbb{P}\left(\left(M^{q}\right)_{t}^{*}>\lambda\right) \lesssim_{X} \mathbb{E}\left\|M_{t}^{d}\right\|, \\
& \lambda \mathbb{P}\left(\left(M^{a}\right)_{t}^{*}>\lambda\right) \lesssim X \mathbb{E}\left\|M_{t}^{d}\right\| .
\end{aligned}
$$

For the proof we will need the following lemmas.

Lemma 4.18. Let $M: \mathbb{R}_{+} \times \Omega \rightarrow \mathbb{R}$ be a local martingale with accessible jumps, $M_{0}=0$ a.s. Then $\left\{M_{\infty}^{*}=0\right\}=\left\{[M]_{\infty}=0\right\}$ up to a negligible set.

Proof. Let $M=M^{c}+M^{q}+M^{a}$ be the canonical decomposition of $M$ (see Subsection 4.1). Then $M^{q}=0$ since $M$ has accessible jumps. By [26, Exercise 17.3] $\left\{\left(M^{c}\right)_{\infty}^{*}=0\right\}=\left\{\left[M^{c}\right]_{\infty}=0\right\}$ up to a negligible set. Let us show the same for $M^{a}$. Let $\tau:=\inf \left\{t \geq 0: \Delta M_{t}^{a} \neq 0\right\}$ be a stopping time. Notice that a.s.

$$
\begin{aligned}
& \{\tau<\infty\} \subset\left\{\sum_{t \geq 0}\left|\Delta M_{t}^{a}\right|>0\right\} \subset\left\{\left(M^{a}\right)_{\infty}^{*}>0\right\}, \\
& \{\tau<\infty\} \subset\left\{\sum_{t \geq 0}\left|\Delta M_{t}^{a}\right|^{2}>0\right\}=\left\{\left[M^{a}\right]_{\infty}>0\right\},
\end{aligned}
$$

so we can redefine $M^{a}:=\left(M^{a}\right)^{\tau}$. By the definition of $\tau$ we have that for each $t \geq 0$ a.s. $\sum_{0 \leq s \leq t}\left|\Delta M_{s}^{a}\right|=\left|\Delta M_{\tau}^{a}\right| \mathbf{1}_{\tau \leq t}$, hence by [54, Theoreme (1-6).3] a.s.

$$
M_{t}^{a}=\Delta M_{\tau}^{a} \mathbf{1}_{\tau \leq t}, \quad t \geq 0 .
$$


IVAN YAROSLAVTSEV

Therefore since $\left[M^{a}\right]_{t}=\left|\Delta M_{\tau}^{a}\right|^{2} \mathbf{1}_{\tau \leq t}$ we have that $\left\{\left(M^{a}\right)_{\infty}^{*}=0\right\}=\left\{\left[M^{a}\right]_{\infty}=0\right\}$ up to a negligible set.

Let us now show the desired. First notice that by [26, Corollary 26.16] a.s.

$$
\left\{[M]_{\infty}=0\right\}=\left\{\left[M^{c}\right]_{\infty}+\left[M^{a}\right]_{\infty}=0\right\}=\left\{\left[M^{c}\right]_{\infty}=0\right\} \cap\left\{\left[M^{a}\right]_{\infty}=0\right\} .
$$

On the other hand a.s.

$$
\begin{aligned}
\left\{M_{\infty}^{*}=0\right\} & =\left\{M_{\infty}^{*}=0\right\} \cap\left\{\Delta M_{t}=0 \forall t \geq 0\right\} \stackrel{(i)}{=}\left\{M_{\infty}^{*}=0\right\} \cap\left\{\left(M^{a}\right)_{\infty}^{*}=0\right\} \\
& \stackrel{(i i)}{=}\left\{\left(M^{c}\right)_{\infty}^{*}=0\right\} \cap\left\{\left(M^{a}\right)_{\infty}^{*}=0\right\} \stackrel{(i i i)}{=}\left\{\left[M^{c}\right]_{\infty}=0\right\} \cap\left\{\left[M^{a}\right]_{\infty}=0\right\} \\
& \stackrel{(i v)}{=}\left\{[M]_{\infty}=0\right\},
\end{aligned}
$$

where (i) holds by (4.17), (ii) follows from the fact that $M^{c}=M-M^{a}$, (iii) follows from the first half of the proof, and finally (iv) follows from (4.18).

Lemma 4.19. Let $M: \mathbb{R}_{+} \times \Omega \rightarrow \mathbb{R}$ be a local martingale, $M=M^{c}+M^{q}+M^{a}$ be the canonical decomposition. Then up to a negligible set

$$
\left\{M_{\infty}^{*}=0\right\}=\left\{\left(M^{c}\right)_{\infty}^{*}=0\right\} \cap\left\{\left(M^{q}\right)_{\infty}^{*}=0\right\} \cap\left\{\left(M^{a}\right)_{\infty}^{*}=0\right\} .
$$

Proof. Let $N:=M^{c}+M^{a}$. First notice that by Lemma 4.18 and 26, Corollary 26.16] a.s.

$$
\begin{aligned}
\left\{N_{\infty}^{*}=0\right\}=\left\{[N]_{\infty}=0\right\} & =\left\{\left[M^{c}\right]_{\infty}+\left[M^{a}\right]_{\infty}=0\right\} \\
& =\left\{\left[M^{c}\right]_{\infty}=0\right\} \cap\left\{\left[M^{a}\right]_{\infty}=0\right\} \\
& =\left\{\left(M^{c}\right)_{\infty}^{*}=0\right\} \cap\left\{\left(M^{a}\right)_{\infty}^{*}=0\right\} .
\end{aligned}
$$

Let $\tau:=\inf \left\{t \geq 0: \Delta M_{t} \neq 0\right\}$ be a stopping time. Then a.s.

$$
\{\tau<\infty\} \subset\left\{M_{\infty}^{*}>0\right\} \subset\left\{N_{\infty}^{*}>0\right\} \cup\left\{\left(M^{q}\right)_{\infty}^{*}>0\right\}
$$

since $M=N+M^{d}$. Let $A=\left\{M_{\infty}^{*}=0\right\} \subset \Omega$. Then $[M]_{\infty}=\left[N+M^{q}\right]_{\infty}=0$ a.s. on $A$, and consequently $[N]_{\infty}=0$ a.s. on $A$ by [26, Corollary 26.16]. Therefore by Lemma $4.18 N_{\infty}^{*}=0$ a.s. on $A$, so $\left(M^{q}\right)_{\infty}^{*}=0$ a.s. on $A$, and therefore by (4.20)

$$
\begin{aligned}
\left\{M_{\infty}^{*}=0\right\}=A & \subset\left\{N_{\infty}^{*}=0\right\} \cap\left\{\left(M^{q}\right)_{\infty}^{*}=0\right\} \\
& =\left\{\left(M^{c}\right)_{\infty}^{*}=0\right\} \cap\left\{\left(M^{q}\right)_{\infty}^{*}=0\right\} \cap\left\{\left(M^{a}\right)_{\infty}^{*}=0\right\} .
\end{aligned}
$$

The converse inclusion follows from the fact that $M=N+M^{q}$ and (4.20).

Lemma 4.20. Let $X$ be a Banach space, $M, N: \mathbb{R}_{+} \times \Omega \rightarrow X$ be local martingales such that $N$ has accessible jumps and $N \stackrel{w}{\ll} M$. Then

$$
\mathbb{P}\left(N_{t}^{*}>0\right) \leq \mathbb{P}\left(M_{t}^{*}>0\right), \quad t \geq 0 .
$$

Proof. (4.21) follows from the fact that $\left\{M_{t}^{*}=0\right\} \subset\left\{N_{t}^{*}=0\right\}$. Let $\left(x_{n}^{*}\right)_{n \geq 0} \subset X^{*}$ be a separating set. Then up to a negligible set

$$
\begin{aligned}
& \left\{M_{t}^{*}=0\right\}=\bigcap_{n \geq 0}\left\{\left(\left\langle M, x_{n}^{*}\right\rangle\right)_{t}^{*}=0\right\}, \\
& \left\{N_{t}^{*}=0\right\}=\bigcap_{n \geq 0}\left\{\left(\left\langle N, x_{n}^{*}\right\rangle\right)_{t}^{*}=0\right\},
\end{aligned}
$$

therefore it is sufficient to consider $X=\mathbb{R}$. Let $M=M^{c}+M^{d}+M^{a}$ be the canonical decomposition of $M$ (see Subsection 4.1). By Lemma 4.19 and 44.20)

$$
\left\{M_{t}^{*}=0\right\} \subset\left\{\left(M^{c}+M^{a}\right)_{t}^{*}=0\right\} .
$$


Moreover, by Lemma 4.18

$$
\begin{aligned}
\left\{\left(M^{c}+M^{a}\right)_{t}^{*}=0\right\} & =\left\{\left[M^{c}+M^{a}\right]_{t}=0\right\} \subset\left\{[M]_{t}=0\right\}, \\
\left\{N_{t}^{*}=0\right\} & =\left\{[N]_{t}=0\right\},
\end{aligned}
$$

and hence since $N \ll M$,

$$
\left\{M_{t}^{*}=0\right\} \subset\left\{[M]_{t}=0\right\} \subset\left\{[N]_{t}=0\right\}=\left\{N_{t}^{*}=0\right\} .
$$

Proof of Theorem 4.17. Without loss of generality assume that $M^{d}$ is an $L^{1}$-martingale and $M_{0}^{d}=0$ a.s. We will divide the proof into two steps.

Step 1. Define a stopping time $\tau=\left\{t \geq 0:\left\|M_{t}^{d}\right\|>\frac{1}{2}\right\}$. In this step we assume that $M^{d}=\left(M^{d}\right)^{\tau}$ (i.e. the martingale stops moving after reaching $\frac{1}{2}$, in particular after the first jump of absolute value bigger than 1). Let $\mu^{M}$ be the random measure defined by (3.3), $\nu^{M}$ be the corresponding compensator (see Subsection 2.4). For each $n \geq 1$ define a stopping time

$$
\tau_{n}=\inf \left\{t \geq 0: \int_{[0, t] \times X}\|x\| \mathbf{1}_{\|x\|>n} \mathrm{~d} \nu^{M^{d}}>1\right\}
$$

and a process $M^{d, n}: \mathbb{R}_{+} \times \Omega \rightarrow X$ in the following way

$$
M_{t}^{d, n}=\left(\left(M^{d}\right)_{t}^{\tau-}+\Delta M_{\tau}^{d} \mathbf{1}_{\left\|\Delta M_{\tau}^{d}\right\| \leq n} \mathbf{1}_{\tau \leq t}+\int_{[0, t] \times X} x \mathbf{1}_{\|x\|>n} \mathrm{~d} \nu^{M^{d}}\right)^{\tau_{n}-}, t \geq 0,
$$

where we define $M^{\sigma-}$ for a stopping time $\sigma$ in the same way as in (2.3). First of all show that $\tau_{n} \rightarrow \infty$ a.s. as $n \rightarrow \infty$. Notice that by due to Subsection 2.4

$$
\begin{aligned}
\mathbb{E} \int_{\mathbb{R}_{+} \times X}\|x\| \mathbf{1}_{\|x\|>1} \mathrm{~d} \nu^{M^{d}} & =\mathbb{E} \int_{\mathbb{R}_{+} \times X}\|x\| \mathbf{1}_{\|x\|>1} \mathrm{~d} \mu^{M^{d}} \leq \mathbb{E}\left\|\Delta M_{\tau}^{d}\right\| \\
& \leq \mathbb{E}\left\|M_{\tau}^{d}\right\|+\mathbb{E}\left\|M_{\tau-}^{d}\right\| \stackrel{(*)}{\leq} \mathbb{E}\left\|M_{\infty}^{d}\right\|+\frac{1}{2} \stackrel{(* *)}{<} \infty,
\end{aligned}
$$

where $(*)$ follows from the fact that $M_{\tau}=M_{\infty}$ and the fact that $\left\|M_{\tau-}\right\| \leq \frac{1}{2}$ a.s., and (**) holds due to the fact that $M$ is an $L^{1}$-martingale. Therefore

$$
\int_{\mathbb{R}_{+} \times X}\|x\| \mathbf{1}_{\|x\|>1} \mathrm{~d} \nu^{M^{d}}<\infty \text { a.s. }
$$

so by the monotone convergence theorem a.s.

$$
\int_{\mathbb{R}_{+} \times X}\|x\| \mathbf{1}_{\|x\|>n} \mathrm{~d} \nu^{M^{d}} \rightarrow 0, \quad n \rightarrow \infty
$$

and hence $\tau_{n} \rightarrow \infty$ as $n \rightarrow \infty$.

We need to show that $M^{d, n}$ is an $L^{\infty}$-martingale for each $n \geq 1$. Clearly $M^{d, n}$ is adapted and càdlàg. It is also a local martingale since it can be rewritten in the following form:

$$
M_{t}^{d, n}=\left(M^{d}\right)_{t}^{\tau_{n}-}-\int_{[0, t] \times X} x \mathbf{1}_{\|x\|>n} \mathbf{1}_{s<\tau_{n}} \mathrm{~d} \bar{\mu}^{M^{d}}, \quad t \geq 0,
$$

where the first term is a martingale by Lemma 2.8. and the second term is a local martingale by Lemma 2.6 and the fact that the process $s \mapsto \mathbf{1}_{s<\tau_{n}}$ is predictable by [26, Theorem 25.14] and the predictability of $\tau_{n}$ (the latter follows from (4.22) 
and the predictability of $\nu^{M^{d}}$, see Subsection 2.4). Moreover, for each fixed $t \geq 0$ we have that a.s.

$$
\begin{aligned}
\left\|M_{t}^{d, n}\right\| & \leq\left\|\left(M^{d}\right)_{t}^{\tau-\wedge \tau_{n}-}\right\|+\left\|\Delta M_{\tau}^{d} \mathbf{1}_{\left\|\Delta M_{\tau}^{d}\right\| \leq n}\right\|+\int_{\left[0, \tau_{n}\right) \times X}\|x\| \mathbf{1}_{\|x\|>n} \nu^{M^{d}} \\
& \leq 1+n+1=n+2 .
\end{aligned}
$$

(Recall that $\tau-\wedge \tau_{n}-:=\left(\tau \wedge \tau_{n}\right)-$, see (3.6) $)$. Therefore $\left(M^{d, n}\right)_{n \geq 1}$ are bounded martingales.

Now let us now show that $M_{\infty}^{d, n} \rightarrow M_{\infty}^{d}$ in $L^{1}(\Omega ; X)$. First, $M_{\infty}^{d, n}=M_{\tau_{n}-}^{d, n}$ a.s., so by the triangle inequality

$$
\mathbb{E}\left\|M_{\infty}^{d}-M_{\infty}^{d, n}\right\| \leq \mathbb{E}\left\|M_{\infty}^{d}-M_{\tau_{n}-}^{d}\right\|+\mathbb{E}\left\|M_{\tau_{n}-}^{d}-M_{\tau_{n}-}^{d, n}\right\| .
$$

Notice that the first term vanishes as $n \rightarrow \infty$ by the fact that $\left\|M_{\infty}^{d}-M_{\tau_{n}-}^{d}\right\| \leq$ $1+\left\|\Delta M_{\tau}\right\|$ a.s., the fact that $\tau_{n} \rightarrow \infty$ a.s., and the dominated convergence theorem. Let us consider the second term:

$$
\begin{aligned}
& \mathbb{E}\left\|M_{\tau_{n}-}^{d}-M_{\tau_{n}-}^{d, n}\right\| \\
& =\mathbb{E}\left\|M_{\tau_{n}-}^{d}-\left(M^{d}\right)_{\tau_{n}-}^{\tau-}-\Delta M_{\tau}^{d} \mathbf{1}_{\left\|\Delta M_{\tau}^{d}\right\| \leq n} \mathbf{1}_{\tau<\tau_{n}}-\int_{\left[0, \tau_{n}\right) \times X} x \mathbf{1}_{\|x\|>n} \mathrm{~d} \nu^{M^{d}}\right\| \\
& =\mathbb{E}\left\|\Delta M_{\tau}^{d} \mathbf{1}_{\tau<\tau_{n}}-\Delta M_{\tau}^{d} \mathbf{1}_{\left\|\Delta M_{\tau}^{d}\right\| \leq n} \mathbf{1}_{\tau<\tau_{n}}-\int_{\left[0, \tau_{n}\right) \times X} x \mathbf{1}_{\|x\|>n} \mathrm{~d} \nu^{M^{d}}\right\| \\
& =\mathbb{E}\left\|\Delta M_{\tau}^{d} \mathbf{1}_{\left\|\Delta M_{\tau}^{d}\right\|>n} \mathbf{1}_{\tau<\tau_{n}}-\int_{\left[0, \tau_{n}\right) \times X} x \mathbf{1}_{\|x\|>n} \mathrm{~d} \nu^{M^{d}}\right\| \\
& =\mathbb{E}\left\|\int_{\left[0, \tau_{n}\right) \times X} x \mathbf{1}_{\|x\|>n} \mathrm{~d} \mu^{M^{d}}-\int_{\left[0, \tau_{n}\right) \times X} x \mathbf{1}_{\|x\|>n} \mathrm{~d} \nu^{M^{d}}\right\| \\
& \leq \mathbb{E} \int_{\left[0, \tau_{n}\right) \times X}\|x\| \mathbf{1}_{\|x\|>n} \mathrm{~d} \mu^{M^{d}}+\mathbb{E} \int_{\left[0, \tau_{n}\right) \times X}\|x\| \mathbf{1}_{\|x\|>n} \mathrm{~d} \nu^{M^{d}} \\
& \stackrel{(*)}{=} 2 \mathbb{E} \int_{\left[0, \tau_{n}\right) \times X}\|x\| \mathbf{1}_{\|x\|>n} \mathrm{~d} \mu^{M^{d} \stackrel{(* *)}{=}} 2 \mathbb{E}\left\|\Delta M_{\tau}^{d}\right\| \mathbf{1}_{\left\|\Delta M_{\tau}^{d}\right\|>n},
\end{aligned}
$$

and the last expression vanishes as $n \rightarrow \infty$ by the monotone convergence theorem. (Notice that $(*)$ follows from the definition of a compensator and from (4.24), while $(* *)$ follows from the fact that $\left\|\Delta M_{t}\right\| \geq 1$ only if $t=\tau$ by the assumptions on $M$.)

Since each of $M^{d, n}$ 's is an $L^{p}$-martingale for each $p \in(1, \infty)$, by Proposition 4.7 for each $n \geq 1$ there exists the Yoeurp decomposition $M^{d, n}=M^{q, n}+M^{a, n}$ of a martingale $M^{d, n}$ into a sum of two purely discontinuous martingales $M^{q, n}, M^{a, n}$ : $\mathbb{R}_{+} \times \Omega \rightarrow X$ such that $M^{q, n}$ is quasi-left continuous, $M^{a, n}$ has accessible jumps, and $M_{0}^{q, n}=M_{0}^{a, n}=0$ a.s. (recall that $M_{0}^{d, n}=0$ a.s.). Fix some $p \in(1, \infty)$. Since an operator $T^{q}$ that maps an $L^{p}$-martingale $M: \mathbb{R}_{+} \times \Omega \rightarrow X$ to its purely discontinuous quasi-left continuous part $M^{q}$ of the canonical decomposition is continuous on $\mathcal{L}\left(\mathcal{M}_{X}^{p}\right)$ by Proposition 4.7. Theorem 4.9 together with Lemma 4.20 yields that for each $m, n \geq 1$ and $K>0$

$$
\begin{aligned}
\mathbb{P}\left(\left(M^{q, n}-M^{q, m}\right)_{\infty}^{*}>K\right) & \lesssim p \frac{1}{K} \mathbb{E}\left\|M_{\infty}^{d, n}-M_{\infty}^{d, m}\right\| \\
& \leq \frac{1}{K}\left(\mathbb{E}\left\|M_{\infty}^{d, n}-M_{\infty}^{d}\right\|+\mathbb{E}\left\|M_{\infty}^{d, m}-M_{\infty}^{d}\right\|\right),
\end{aligned}
$$


so $\left(M^{q, n}\right)_{n \geq 1}$ is a Cauchy sequence in the ucp topology. By Proposition 2.2 it has a càdlàg adapted limit. Denote this limit by $M^{q}$. Let us show that $M^{q}$ is a purely discontinuous quasi-left continuous local martingale. Let $\sigma$ be a predictable time. Then $\Delta M_{\sigma}^{q, n}=0$ a.s., and for any $t \geq 0$ a.s.

$$
\begin{aligned}
\sup _{0 \leq s \leq t}\left\|M_{s}^{q, n}-M_{s}^{q}\right\| & \geq \mathbf{1}_{\sigma \leq t} \sup _{0 \leq s \leq \sigma}\left\|M_{s}^{q, n}-M_{s}^{q}\right\| \\
& \geq \mathbf{1}_{\sigma \leq t}\left(\sup _{m \geq 1}\left\|M_{0 \vee \sigma-\frac{1}{m}}^{q, n}-M_{0 \vee \sigma-\frac{1}{m}}^{q}\right\| \vee\left\|M_{\sigma}^{q, n}-M_{\sigma}^{q}\right\|\right) \\
& \geq \frac{1}{2} \mathbf{1}_{\sigma \leq t}\left(\limsup _{m \geq 1}\left\|M_{0 \vee \sigma-\frac{1}{m}}^{q, n}-M_{0 \vee \sigma-\frac{1}{m}}^{q}\right\|+\left\|M_{\sigma}^{q, n}-M_{\sigma}^{q}\right\|\right) \\
& =\frac{1}{2} \mathbf{1}_{\sigma \leq t}\left(\left\|M_{\sigma-}^{q, n}-M_{\sigma-}^{q}\right\|+\left\|M_{\sigma}^{q, n}-M_{\sigma}^{q}\right\|\right) \\
& \stackrel{(*)}{\geq} \frac{1}{2} \mathbf{1}_{\sigma \leq t}\left\|M_{\sigma-}^{q, n}-M_{\sigma-}^{q}-M_{\sigma}^{q, n}+M_{\sigma}^{q}\right\| \\
& \geq \frac{1}{2} \mathbf{1}_{\sigma \leq t}\left\|\Delta M_{\sigma-}^{q}-\Delta M_{\sigma}^{q, n}\right\|=\frac{1}{2} \mathbf{1}_{\sigma \leq t}\left\|\Delta M_{\sigma-}^{q}\right\|,
\end{aligned}
$$

where $(*)$ follows from the triangle inequality. Since

$$
\mathbb{P}-\lim _{n \rightarrow \infty} \sup _{0 \leq s \leq t}\left\|M_{s}^{q, n}-M_{s}^{q}\right\|=0,
$$

we have that for each $t \geq 0$

$$
\mathbb{P}-\lim _{n \rightarrow \infty} \mathbf{1}_{\sigma \leq t}\left\|\Delta M_{\sigma-}^{q}\right\|=0 .
$$

But the expression under the limit in probability does not depend on $n$. Hence $\mathbf{1}_{\sigma \leq t}\left\|\Delta M_{\sigma-}^{q}\right\|=0$ a.s. By letting $t \rightarrow \infty$ we get that a.s. $\left\|\Delta M_{\sigma}^{q}\right\|=0$, and since $\sigma$ was arbitrary predictable, $M^{q}$ is quasi-left continuous.

Let now $\sigma$ be a totally inaccessible stopping time. Let us show that a.s.

$$
\Delta M_{\sigma}^{q}=\Delta M_{\sigma}^{d} .
$$

First notice that for each fixed $m \geq n \geq 1$

$$
\begin{aligned}
& \Delta M_{\sigma}^{q, m} \mathbf{1}_{\sigma<\tau \wedge \tau_{n}} \stackrel{(*)}{=} \Delta M_{\sigma}^{d, m} \mathbf{1}_{\sigma<\tau \wedge \tau_{n}} \stackrel{(* *)}{=} \Delta M_{\sigma}^{d} \mathbf{1}_{\sigma<\tau \wedge \tau_{n}}, \\
& \Delta M_{\sigma}^{q, m} \mathbf{1}_{\sigma=\tau<\tau_{n}} \mathbf{1}_{\left\|\Delta M_{\tau}^{d}\right\| \leq n} \stackrel{(*)}{=} \Delta M_{\sigma}^{d, m} \mathbf{1}_{\sigma=\tau<\tau_{n}} \mathbf{1}_{\left\|\Delta M_{\tau}^{d}\right\| \leq n} \stackrel{(* *)}{=} \Delta M_{\sigma}^{d} \mathbf{1}_{\sigma=\tau<\tau_{n}} \mathbf{1}_{\left\|\Delta M_{\tau}^{d}\right\| \leq n},
\end{aligned}
$$

where $(*)$ follows from Remark 4.6, and $(* *)$ follows from the definition (4.23) of $M^{d, m}$ and Lemma 2.7. Therefore by (4.25) applied for our $\sigma$ a.s. for each $n \geq 1$

$$
\begin{aligned}
& \Delta M_{\sigma}^{d} \mathbf{1}_{\sigma<\tau \wedge \tau_{n}}=\Delta M_{\sigma}^{q} \mathbf{1}_{\sigma<\tau \wedge \tau_{n}}, \\
& \Delta M_{\sigma}^{d} \mathbf{1}_{\sigma=\tau<\tau_{n}} \mathbf{1}_{\left\|\Delta M_{\tau}^{d}\right\| \leq n}=\Delta M_{\sigma}^{q} \mathbf{1}_{\sigma=\tau<\tau_{n}} \mathbf{1}_{\left\|\Delta M_{\tau}^{d}\right\| \leq n} .
\end{aligned}
$$

By letting $n \rightarrow \infty$ we get (4.26).

Let us show that $M^{q}$ is locally integrable. For each $l \geq 1$ set $\rho_{l}:=\inf \{t \geq 0$ : $\left.\left\|M_{t}^{q}\right\| \geq l\right\}$. Then a.s. for each $t \geq 0$

$$
\begin{aligned}
\left\|\left(M^{q}\right)_{t}^{\rho_{l}}\right\| \leq\left\|\left(M^{q}\right)_{t-}^{\rho_{l}}\right\|+\left\|\Delta\left(M^{q}\right)_{t}^{\rho_{l}}\right\| & \leq l+\left\|\Delta\left(M^{q}\right)_{t}^{\rho_{l}}\right\| \mathbf{1}_{t=\tau}+\left\|\Delta\left(M^{q}\right)_{t}^{\rho_{l}}\right\| \mathbf{1}_{t<\tau} \\
& \leq l+\left\|\Delta M_{\tau}^{d}\right\|+1 .
\end{aligned}
$$

Therefore

$$
\mathbb{E}\left\|\left(M^{q}\right)_{t}^{\rho_{l}}\right\| \leq l+1+\mathbb{E}\left\|\Delta M_{\tau}^{d}\right\|<\infty,
$$


where $\mathbb{E}\left\|\Delta M_{\tau}\right\|<\infty$ by (4.24). Since $M^{q}$ is càdlàg, by [44, Problem V.1] we have that $\rho_{l} \rightarrow \infty$ as $l \rightarrow \infty$, so $M^{q}$ is locally integrable.

Now let us show that $M^{q}$ is a local martingale. Let $\left(M^{q, n_{k}}\right)_{k \geq 1}$ be a subsequence of $\left(M^{q, n}\right)_{n \geq 1}$ such that $M^{q, n_{k}} \rightarrow M^{q}$ uniformly on compacts a.s. (existence of such a subsequence can be shown e.g. as in the proof of [45, Theorem 62]). It is sufficient to show that $M^{\rho_{l} \wedge \tau_{n_{k}}-}$ is a local martingale for each $l, k \geq 1$ since $\rho_{l} \rightarrow \infty$ and $\tau_{n_{k}} \rightarrow \infty$ a.s. as $l, k \rightarrow \infty$. Fix $K>0$. Then by (4.27) and (4.28) for each $k \geq K$ we have that a.s. for each $t \geq 0$

$$
\Delta\left(M^{q, n_{k}}\right)_{t}^{\tau_{n_{K}}-\wedge \tau-}=\Delta\left(M^{q}\right)_{t}^{\tau_{n_{K}}-\wedge \tau-} .
$$

Therefore by Lemma 2.3 there exists a continuous adapted process $N: \mathbb{R}_{+} \times \Omega \rightarrow$ $\mathbb{R}_{+}$such that a.s.

$$
N_{t}=\sup _{k \geq K}\left\|\left(M^{q, n_{k}}\right)_{t}^{\tau_{n_{K}}-\wedge \tau-}-\left(M^{q}\right)_{t}^{\tau_{n_{K}}-\wedge \tau-}\right\|, \quad t \geq 0 .
$$

Now for each $j \geq 1$ define a stopping time $\sigma_{j}=\inf \left\{t \geq 0: N_{t} \geq j\right\}$. Fix $j \geq 1$. Then for each $t \geq 0$ we have that for any $k \geq K$ a.s.

$$
\left\|\left(M^{q, n_{k}}\right)_{t}^{\rho_{l} \wedge \tau_{n_{K}}-\wedge \sigma_{j}}-\left(M^{q}\right)_{t}^{\rho_{l} \wedge \tau_{n_{K}}-\wedge \sigma_{j}}\right\| \leq j+l+2\left\|\Delta M_{\tau}^{d}\right\|
$$

and that $\left(M^{q, n_{k}}\right)_{t}^{\rho_{l} \wedge \tau_{n_{K}}-\wedge \sigma_{j}}-\left(M^{q}\right)_{t}^{\rho_{l} \wedge \tau_{n_{K}}-\wedge \sigma_{j}} \rightarrow 0$ a.s. as $k \rightarrow \infty$. Hence by the dominated convergence theory

$$
\left(M^{q, n_{k}}\right)_{t}^{\rho_{l} \wedge \tau_{n_{K}}-\wedge \sigma_{j}} \rightarrow\left(M^{q}\right)_{t}^{\rho_{l} \wedge \tau_{n_{K}}-\wedge \sigma_{j}} \text { in } L^{1}(\Omega ; X) \text { as } k \rightarrow \infty .
$$

Consequently, $\left(\left(M^{q}\right)_{t}^{\rho_{l} \wedge \tau_{n_{K}}-\wedge \sigma_{j}}\right)_{t>0}$ is an $L^{1}$-martingale, which is moreover purely discontinuous by Lemma 4.16. By letting $l, K, j \rightarrow \infty$ we get that $M^{q}$ is a purely discontinuous quasi-left continuous local martingale.

$M^{a}$ can be constructed in the same way. The identity $M^{d}=M^{q}+M^{a}$ follows from the following limiting argument:

$$
\begin{aligned}
& M^{d}=u c p-\lim _{n \rightarrow \infty} M^{d, n}, \\
& M^{q}=u c p-\lim _{n \rightarrow \infty} M^{q, n}, \\
& M^{a}=u c p-\lim _{n \rightarrow \infty} M^{a, n},
\end{aligned}
$$

and the fact that $M^{d, n}=M^{q, n}+M^{a, n}$ for each $n \geq 1$.

Step 2. For a general martingale $M^{d}$ we construct a sequence of stopping times $\tau_{n}=\inf \left\{t \geq 0:\left\|M_{t}^{d}\right\| \geq \frac{n}{2}\right\}$. For each $M^{d, n}:=\left(M^{d}\right)^{\tau_{n}}$ we construct the corresponding $M^{q, n}$ by Step 1 . Then for each $m \geq n \geq 1$ we get that $\left(M^{q, n}\right)^{\tau_{m}}=M^{q, m}$ since for any $x^{*} \in X^{*}$ a.s.

$$
\left\langle\left(M^{q, n}\right)^{\tau_{m}}, x^{*}\right\rangle=\left\langle M^{q, m}, x^{*}\right\rangle
$$

due to the uniqueness of the Yoeurp decomposition in the real-valued case. Then we just set $M_{0}^{q}:=0$ and

$$
M_{t}^{q}:=\sum_{n \geq 1} M_{t}^{q, n} \mathbf{1}_{t \in\left(\tau_{n-1}, \tau_{n}\right]}, \quad t \geq 0,
$$

where $\tau_{0} \equiv 0$. The obtained $M^{q}$ will be the desired purely discontinuous quasi-left continuous local martingale.

We can construct $M^{a}$ in the same way and show that then $M^{d}=M^{q}+M^{a}$ similarly to how it was shown in step 1. 
The uniqueness of the decomposition follows from Remark 4.4, while (4.16) follows analogously (4.14).

Proof of Theorem 4.8 (sufficiency of UMD and (4.2)). Sufficiency of the UMD property follows from Theorem 4.15 and Theorem 4.17, while (4.2) follows in the same way as (4.14) and (4.16).

4.4. Necessity of the UMD property. In the current subsection we show that the UMD property is necessary in Theorem 4.15] and Theorem 4.17, and hence it is necessary for the canonical decomposition of a local martingale.

Theorem 4.21. Let $X$ be a Banach space that does not have the UMD property. Then there exists a filtration $\mathbb{F}=\left(\mathcal{F}_{t}\right)_{t \geq 0}$ and an $\mathbb{F}$-martingale $M: \mathbb{R}_{+} \times \Omega \rightarrow X$ such that $M$ provides neither the Meyer-Yoeurp nor the canonical decomposition.

For the proof we will need the following lemma which is a modification of the statements from p. 1001 and p. 1004 of [6]. Recall that if $\left(f_{n}\right)_{n>0}$ is an $X$-valued martingale, the we define $d f_{n}:=f_{n}-f_{n-1}$ for $n \geq 1$ and $d f_{0}:=f_{0}$.

Lemma 4.22. Let $X$ be a Banach space. Then $X$ is a UMD Banach space if and only if there exists a constant $C>0$ such that for any $X$-valued discrete martingale $\left(f_{n}\right)_{n \geq 0}$, for any $\{0,1\}$-valued sequence $\left(a_{n}\right)_{n \geq 0}$ one has that

$$
g_{\infty}^{*}>1 \text { a.s. } \Longrightarrow \mathbb{E}\left\|f_{\infty}\right\|>C,
$$

where $\left(g_{n}\right)_{n \geq 0}$ is an $X$-valued discrete martingale such that $d g_{n}=a_{n} d f_{n}$ for each $n \geq 0, g_{\infty}^{*}:=\sup _{n \geq 0}\left\|g_{n}\right\|$.

Proof. One needs to modify [6, Theorem 2.1] in such a way that $d g_{n}=a_{n} d f_{n}$ for some $a_{n} \in\{0,1\}$ for each $n \geq 0$. Then the proof is the same, and the desired statement follows from the equivalence of [6, (2.3)] and $[6,(2.4)]$.

For the next corollary we will need to define a Rademacher random variable and a Paley-Walsh martingale.

Definition 4.23 (Rademacher random variable). Let $\xi: \Omega \rightarrow \mathbb{R}$ be a random variable. Then $\xi$ has the Rademacher distribution (or simply $\xi$ is Rademacher) if $\mathbb{P}(\xi=1)=\mathbb{P}(\xi=-1)=\frac{1}{2}$.

Definition 4.24 (Paley-Walsh martingale). Let $X$ be a Banach space. A discrete $X$-valued martingale $\left(f_{n}\right)_{n \geq 0}$ is called a Paley-Walsh martingale if there exist a sequence of independent Rademacher random variables $\left(r_{n}\right)_{n>1}$, a function $\phi_{n}$ : $\{-1,1\}^{n-1} \rightarrow X$ for each $n \geq 2$ and $\phi_{1} \in X$ such that $d f_{n}=r_{n} \phi_{n}\left(r_{1}, \ldots, r_{n-1}\right)$ for each $n \geq 2, d f_{1}=r_{1} \phi_{1}$, and $f_{0}$ is a constant a.s.

Corollary 4.25. Let $X$ be a Banach space that does not have the UMD property. Then there exists an $X$-valued Paley-Walsh $L^{1}$-martingale $\left(f_{n}\right)_{n \geq 0}$ and $a\{0,1\}$ valued sequence $\left(a_{n}\right)_{n \geq 0}$ such that $\mathbb{P}\left(g_{\infty}^{*}=\infty\right)=1$, where $\left(g_{n}\right)_{n \geq 0}$ is an $X$-valued martingale such that $\bar{d} g_{n}=a_{n} d f_{n}$ for each $n \geq 0$.

Proof. Without loss of generality all the martingales used below are Paley-Walsh (see [23, Theorem 3.6.1]), so the resulting martingale will be Paley-Walsh as well. By Lemma 4.22 we can find $N_{1}>0$, an $X$-valued martingale $f^{1}=\left(f_{n}^{1}\right)_{n=0}^{N_{1}}$ and a $\{0,1\}$-valued sequence $\left(a_{n}^{1}\right)_{n=0}^{N_{1}}$ such that $\mathbb{E}\left\|f_{N_{1}}^{1}\right\|<\frac{1}{2}$ and

$$
\mathbb{P}\left(\left(g^{1}\right)_{N_{1}}^{*}>1\right)>\frac{1}{2}
$$


where $g^{1}=\left(g_{n}^{1}\right)_{n=0}^{N_{1}}$ is such that $d g_{n}^{1}=a_{n}^{1} d f_{n}^{1}$ for each $n=0, \ldots, N_{1}$. Now inductively for each $k>1$ we find $N_{k}>0$ and an $X$-valued Paley-Walsh martingale $f^{k}=\left(f_{n}^{k}\right)_{n=0}^{N_{k}}$ independent of $f^{1}, \ldots, f^{k-1}$ such that $\mathbb{E}\left\|f_{N_{k}}^{k}\right\|<\frac{1}{2^{k}}$ and

$$
\mathbb{P}\left(\left(g^{k}\right)_{N_{k}}^{*}>2 C_{k}\right)>1-\frac{1}{2^{k}}
$$

where $g^{k}=\left(g_{n}^{k}\right)_{n=0}^{N_{1}}$ is such that $d g_{n}^{k}=a_{n}^{k} d f_{n}^{k}$ for each $n=0, \ldots, N_{k}$, and $C_{k}>2^{k}$ is such that

$$
\mathbb{P}\left(\left(g^{1}\right)_{N_{1}}^{*}+\ldots+\left(g^{k-1}\right)_{N_{k-1}}^{*}>C_{k}\right)<\frac{1}{2^{k}} .
$$

Without loss of generality assume that $f_{0}^{k}=0$ a.s. for each $k \geq 1$. Now construct a martingale $\left(f_{n}\right)_{n \geq 0}$ and a $\{0,1\}$-valued sequence $\left(a_{n}\right)_{n \geq 0}$ in the following way: $f_{0}=a_{0}=0$ a.s., $d f_{n}=d f_{m}^{k}$ and $a_{n}=a_{m}^{k}$ if $n=N_{1}+\cdots+N_{k-1}+m$ for some $k \geq 1$ and $1 \leq m \leq N_{k}$. Then $\left(f_{n}\right)_{n \geq 0}$ is well-defined,

$$
\lim _{n \rightarrow \infty} \mathbb{E}\left\|f_{n}\right\|=\mathbb{E}\left\|f_{\infty}\right\| \leq \sum_{k \geq 1} \mathbb{E}\left\|f_{N_{k}}^{k}\right\| \leq 1
$$

by the triangle inequality, and for an $X$-valued martingale $\left(g_{n}\right)_{n \geq 0}$ with $d g_{n}=a_{n} d f_{n}$ for each $n \geq 0$, for each $k \geq 2$

$\mathbb{P}\left(g_{N_{1}+\cdots+N_{k}}^{*}>C_{k}\right) \geq \mathbb{P}\left(\left(g^{k}\right)_{N_{k}}^{*}>2 C_{k},\left(g^{1}\right)_{N_{1}}^{*}+\ldots+\left(g^{k-1}\right)_{N_{k-1}}^{*} \leq C_{k}\right)>1-\frac{1}{2^{k-1}}$, hence $g_{\infty}^{*}=\infty$ a.s.

Proof of Theorem 4.21. By Corollary 4.25 we can construct a discrete filtration $\mathbb{G}=\left(\mathcal{G}_{n}\right)_{n \geq 0}$ and an $X$-valued $L^{1}$-integrable Paley-Walsh $\mathbb{G}$-martingale $\left(f_{n}\right)_{n \geq 0}$ such that

$$
\mathbb{E}\left\|f_{\infty}\right\|=\lim _{n \rightarrow \infty} \mathbb{E}\left\|f_{n}\right\| \leq 1,
$$

and such that there exists $\{0,1\}$-valued sequence $\left(a_{n}\right)_{n \geq 0}$ so that

$$
\mathbb{P}\left(g_{\infty}^{*}=\infty\right)=1,
$$

where $\left(g_{n}\right)_{n \geq 0}$ is an $X$-valued martingale with $d g_{n}=a_{n} d f_{n}$ for each $n \geq 0$.

Since $\left(f_{n}\right)_{n \geq 0}$ is Paley-Walsh, there exist a sequence $\left(r_{n}\right)_{n \geq 0}$ of independent Rademacher variables, a sequence of functions $\left(\phi_{n}\right)_{n \geq 1}$ with $\phi_{1} \in X$ and $\phi_{n}$ : $\{-1,1\}^{n-1} \rightarrow X$ for each $n \geq 2$, so that $d f_{n}=r_{n} \phi_{n}\left(r_{1}, \ldots, r_{n-1}\right)$ a.s. for each $n \geq 1$.

Now our goal is to construct a continuous-time $X$-valued martingale $M$ which does not have the Meyer-Yoeurp decomposition (and hence the canonical decomposition) using $\left(f_{n}\right)_{n \geq 0}$. Let us first construct a filtration $\mathbb{F}=\left(\mathcal{F}_{t}\right)_{t \geq 0}$ on $\mathbb{R}_{+}$in the following way. By [51, Subsection 3.2] for each $n \geq 0$ we can find a continuous martingale $M^{n}:\left[0, \frac{1}{2^{n+1}}\right] \times \Omega \rightarrow \mathbb{R}$ with a symmetric distribution such that $M_{0}^{n}=0$ a.s., $\left|M_{\frac{1}{2^{n+1}}}^{n}\right| \leq 1$ a.s.,

$$
\mathbb{P}\left(M_{\frac{1}{2^{n+1}}}^{n}=0\right)=0
$$

and

$$
\mathbb{P}\left(M_{\frac{1}{2^{n+1}}}^{n} \neq \operatorname{sign} M_{\frac{1}{2^{n+1}}}^{n}\right)<\frac{1}{2^{n}\left(\left\|\phi_{n}\right\|_{\infty}+1\right)} .
$$


Let $\left(\tilde{r}_{n}\right)_{n \geq 0}$ be a sequence of independent Rademacher random variables. Without loss of generality assume that all $\left(\tilde{r}_{n}\right)_{n \geq 0}$ and $\left(M^{n}\right)_{n \geq 0}$ are independent. Then set $\mathcal{F}_{0}$ to be the $\sigma$-algebra generated by all negligible sets, and set

$$
\mathcal{F}_{t}:= \begin{cases}\mathcal{F}_{1-\frac{1}{2^{n}}}, & t \in\left(1-\frac{1}{2^{n}}, 1-\frac{1}{2^{n+1}}\right), a_{n}=0, n \geq 0, \\ \sigma\left(\mathcal{F}_{1-\frac{1}{2^{n}}}, \tilde{r}_{n}\right), & t=1-\frac{1}{2^{n+1}}, a_{n}=0, n \geq 0, \\ \sigma\left(\mathcal{F}_{1-\frac{1}{2^{n}}},\left(M_{s}^{n}\right)_{s \in\left[0, t-1-\frac{1}{2^{n}}\right]}\right), & t \in\left(1-\frac{1}{2^{n}}, 1-\frac{1}{2^{n+1}}\right], a_{n}=1, n \geq 0, \\ \sigma\left(\mathcal{F}_{s}: s \in[0,1)\right), & t \geq 1 .\end{cases}
$$

Let $\left(\sigma_{n}\right)_{n \geq 0}$ be a sequence of independent Rademacher variables such that $\sigma_{n}=\tilde{r}_{n}$ if $a_{n}=0$ and $\sigma_{n}=\operatorname{sign} M_{\frac{1}{2^{n+1}}}^{n}$ if $a_{n}=1$ (in the latter case $\sigma_{n}$ has the Rademacher distribution by (4.30) and the fact that $M_{\frac{1}{2^{n+1}}}^{n}$ is symmetric). Now construct $M$ : $\mathbb{R}_{+} \times \Omega \rightarrow X$ in the following way:

$$
M_{t}= \begin{cases}0, & t=0, \\ M_{1-\frac{1}{2^{n}}}, & t \in\left(1-\frac{1}{2^{n}}, 1-\frac{1}{2^{n+1}}\right), a_{n}=0, n \geq 0, \\ M_{1-\frac{1}{2^{n}}}+\sigma_{n} \phi_{n}\left(\sigma_{1}, \ldots, \sigma_{n-1}\right), & t=1-\frac{1}{2^{n+1}}, a_{n}=0, n \geq 0, \\ M_{1-\frac{1}{2^{n}}}+M_{t-1-\frac{1}{2^{n}}}^{n} \phi_{n}\left(\sigma_{1}, \ldots, \sigma_{n-1}\right), & t \in\left(1-\frac{1}{2^{n}}, 1-\frac{1}{2^{n+1}}\right], a_{n}=1, n \geq 0, \\ \lim _{n \rightarrow \infty} M_{1-\frac{1}{2^{n}}}, & t \geq 1 .\end{cases}
$$

First we show that $\lim _{n \rightarrow \infty} M_{1-\frac{1}{2^{n}}}$ exists a.s., hence $M$ is well-defined. By 23 , Theorem 3.3.8] it is sufficient to show that there exists $\xi \in L^{1}(\Omega ; X)$ such that $M_{1-\frac{1}{2^{n}}}=\mathbb{E}\left(\xi \mid \mathcal{F}_{1-\frac{1}{2^{n}}}\right)$ for all $n \geq 1$. Notice that $\left(M_{1-\frac{1}{2^{n}}}\right)_{n \geq 0}$ is a martingale since $M_{1-\frac{1}{2^{n+1}}}-M_{1-\frac{1}{2^{n}}}$ equals either $\sigma_{n} \phi_{n}\left(\sigma_{1}, \ldots, \sigma_{n-1}\right)$ (if $a_{n}=0$ ) or $M_{\frac{1}{2^{n+1}}}^{n} \phi_{n}\left(\sigma_{1}, \ldots, \sigma_{n-1}\right)$ (if $a_{n}=1$ ). Both random variables are bounded, and in both cases the conditional expectation with respect to $\mathcal{F}_{1-\frac{1}{2^{n}}}$ gives zero. Now let us show integrability. Let $\left(\tilde{f}_{n}\right)_{n \geq 0}$ be an $X$-valued martingale such that $\tilde{f}_{0}=0$ a.s. and

$$
d \tilde{f}_{n}=\sigma_{n} \phi_{n}\left(\sigma_{1}, \ldots, \sigma_{n-1}\right), \quad n \geq 1 .
$$

Then $\left(\tilde{f}_{n}\right)_{n \geq 0}$ has the same distribution as $\left(f_{n}\right)_{n \geq 0}$, so it is $L^{1}$-integrable. Now fix $n \geq 1$ and let us estimate $\mathbb{E}\left\|\tilde{f}_{n}-M_{1-\frac{1}{2^{n}}}\right\|$ :

$$
\begin{aligned}
\mathbb{E}\left\|\tilde{f}_{n}-M_{1-\frac{1}{2^{n}}}\right\| \stackrel{(i)}{=} & \mathbb{E} \| \sum_{k=1}^{n} \sigma_{k} \phi_{k}\left(\sigma_{1}, \ldots, \sigma_{k-1}\right) \\
& -\sum_{k=1}^{n}\left(\sigma_{k} \phi_{k}\left(\sigma_{1}, \ldots, \sigma_{k-1}\right) \mathbf{1}_{a_{k}=0}+M_{\frac{1}{2^{k+1}}}^{k} \phi_{k}\left(\sigma_{1}, \ldots, \sigma_{k-1}\right) \mathbf{1}_{a_{k}=1}\right) \| \\
= & \mathbb{E}\left\|\sum_{k=1}^{n}\left(\sigma_{k}-M_{\frac{1}{2^{k+1}}}^{k}\right) \phi_{k}\left(\sigma_{1}, \ldots, \sigma_{k-1}\right) \mathbf{1}_{a_{k}=1}\right\| \\
& \stackrel{(i i)}{\leq} \sum_{k=1}^{n} \mathbb{E}\left\|\left(\sigma_{k}-M_{\frac{1}{2^{k+1}}}^{k}\right) \phi_{k}\left(\sigma_{1}, \ldots, \sigma_{k-1}\right)\right\| \\
& \stackrel{(i i i)}{\leq} 2 \sum_{k=1}^{n} \mathbb{P}\left(\sigma_{k} \neq M_{\frac{1}{2^{k+1}}}^{k}\right)\left\|\phi_{k}\right\|_{\infty} \stackrel{(i v)}{\leq} 2 \sum_{k=1}^{n} \frac{1}{2^{k}} \leq 2,
\end{aligned}
$$


where $(i)$ follows from (4.33) and the definition of $M$ from (4.32), (ii) holds by the triangle inequality, (iii) follows from the fact that a.s. for each $n \geq 1$

$$
\left|\sigma_{n}-M_{\frac{1}{2^{n+1}}}^{n}\right| \leq\left|\sigma_{n}\right|+\left|M_{\frac{1}{2^{n+1}}}^{n}\right| \leq 2 ;
$$

finally, (iv) follows from (4.31). Let us show that there exists $\mathcal{F}_{1}$-measurable $\xi \in L^{1}(\Omega ; X)$ such that $M_{1-\frac{1}{2^{n}}}=\mathbb{E}\left(\xi \mid \mathcal{F}_{1-\frac{1}{2^{n}}}\right)$ for each $n \geq 1$. First notice that $\mathbb{E}\left(\tilde{f}_{\infty} \mid \mathcal{F}_{1-\frac{1}{2^{n}}}\right)=\tilde{f}_{n}$ for each $n \geq 1$. Moreover, by (4.34) the series

$$
\eta:=\sum_{k=1}^{\infty}\left(\sigma_{k}-M_{\frac{1}{2^{k+1}}}^{k}\right) \phi_{k}\left(\sigma_{1}, \ldots, \sigma_{k-1}\right) \mathbf{1}_{a_{k}=1}
$$

converges in $L^{1}(\Omega ; X)$. Therefore, if we define $\xi:=\tilde{f}_{\infty}-\eta$, then

$$
\begin{aligned}
\mathbb{E}\left(\xi \mid \mathcal{F}_{1-\frac{1}{2^{n}}}\right) & =\mathbb{E}\left(\tilde{f}_{\infty}-\eta \mid \mathcal{F}_{1-\frac{1}{2^{n}}}\right) \\
& =\tilde{f}_{n}-\mathbb{E}\left(\sum_{k=1}^{\infty}\left(\sigma_{k}-M_{\frac{1}{2^{k+1}}}^{k}\right) \phi_{k}\left(\sigma_{1}, \ldots, \sigma_{k-1}\right) \mathbf{1}_{a_{k}=1} \mid \mathcal{F}_{1-\frac{1}{2^{n}}}\right) \\
& =\tilde{f}_{n}-\sum_{k=1}^{\infty} \mathbb{E}\left(\left(\sigma_{k}-M_{\frac{1}{2^{k+1}}}^{k}\right) \phi_{k}\left(\sigma_{1}, \ldots, \sigma_{k-1}\right) \mathbf{1}_{a_{k}=1} \mid \mathcal{F}_{1-\frac{1}{2^{n}}}\right) \\
& =\tilde{f}_{n}-\sum_{k=1}^{n}\left(\sigma_{k}-M_{\frac{1}{2^{k+1}}}^{k}\right) \phi_{k}\left(\sigma_{1}, \ldots, \sigma_{k-1}\right) \mathbf{1}_{a_{k}=1}=M_{1-\frac{1}{2^{n}}}
\end{aligned}
$$

so one has an a.s. convergence by the martingale convergence theorem 23, Theorem 3.3.8].

Now let us show that $M$ is a martingale that does not have the Meyer-Yoeurp decomposition. Assume the contrary: let $M=M^{d}+M^{c}$ be the Meyer-Yoeurp decomposition. Then one can show that for each $n \geq 1$

$$
\begin{aligned}
& M_{1-\frac{1}{2^{n}}}^{d}=\sum_{k=1}^{n} \sigma_{k} \phi_{k}\left(\sigma_{1}, \ldots, \sigma_{k-1}\right) \mathbf{1}_{a_{k}=0}, \\
& M_{1-\frac{1}{2^{n}}}^{c}=\sum_{k=1}^{n} M_{\frac{1}{2^{k+1}}}^{k} \phi_{k}\left(\sigma_{1}, \ldots, \sigma_{k-1}\right) \mathbf{1}_{a_{k}=1},
\end{aligned}
$$

by applying $x^{*} \in X^{*}$ and showing that the corresponding processes $\left\langle M_{1-\frac{1}{2^{n}}}^{d}, x^{*}\right\rangle$ and $\left\langle M_{1-\frac{1}{2^{n}}}^{c}, x^{*}\right\rangle$ are purely discontinuous and continuous local martingales respectively (see Remark 4.2). Now let us show that $M^{c}$ is not an $X$-valued local martingale. If it is a local martingale, then

$$
\mathbb{P}\left(\left(M^{c}\right)_{\infty}^{*}=\infty\right)=\mathbb{P}\left(\left(M^{c}\right)_{1}^{*}=\infty\right)=0 .
$$

since $M^{c}$ as a local martingale should have càdlàg paths (even continuous since $M^{c}$ assume to be continuous). But for each fixed $n \geq 1$

$$
\mathbb{P}\left(\left(M^{c}\right)_{1}^{*}=\infty\right)=\mathbb{P}\left(\left(M^{c}-M_{\frac{1}{2^{n}}}^{c}\right)_{1}^{*}=\infty\right) \geq \mathbb{P}\left(\left(\tilde{g}-\tilde{g}_{n}\right)_{\infty}^{*}=\left(M^{c}-M_{\frac{1}{2^{n}}}^{c}\right)_{1}^{*}\right)
$$

where $\left(\tilde{g}_{n}\right)_{n \geq 0}$ is an $X$-valued martingale such that $d \tilde{g}_{n}=a_{n} d \tilde{f}_{n}$ a.s. for each $n \geq 0$, and hence by the construction in Lemma $4.22 \tilde{g}_{\infty}^{*}=\infty$ a.s. Further,

$$
\mathbb{P}\left(\left(\tilde{g}-\tilde{g}_{n}\right)_{\infty}^{*}=\left(M^{c}-M_{\frac{1}{2^{n}}}^{c}\right)_{1}^{*}\right)=1-\mathbb{P}\left(\left(\tilde{g}-\tilde{g}_{n}\right)_{\infty}^{*} \neq\left(M^{c}-M_{\frac{1}{2^{n}}}^{c}\right)_{1}^{*}\right)
$$




$$
\begin{aligned}
& \geq 1-\sum_{k=n}^{\infty} \mathbf{1}_{a_{k}=1} \mathbb{P}\left(M_{\frac{1}{2^{k+1}}}^{k} \neq \sigma_{k}\right) \\
& \geq 1-\sum_{k=n}^{\infty} \mathbb{P}\left(M_{\frac{1}{2}^{k+1}}^{k} \neq \operatorname{sign} M_{\frac{1}{2^{k+1}}}^{k}\right) \\
& \stackrel{(*)}{\geq} 1-\frac{1}{2^{n-1}},
\end{aligned}
$$

where $(*)$ follows from (4.31). Since $n$ was arbitrary, $\left(M^{c}\right)_{1}^{*}=\left(M^{c}\right)_{\infty}^{*}=\infty$ a.s., so $M^{c}$ can not be a local martingale.

Proof of Theorem 4.8 (necessity of UMD). Necessity of the UMD property follows from Theorem 4.21 .

Remark 4.26. One can also show that existence of the Yoeurp decomposition of an arbitrary $X$-valued purely discontinuous local martingale is equivalent to the UMD property. We will not repeat the argument here, but just notice that one needs to modify the proof of Theorem 4.21 in a way which was demonstrated in 51, Subsection 3.2].

Remark 4.27. The reader might assume that one can weaken the Meyer-Yoeurp decomposition and consider a decomposition of an $X$-valued local martingale $M$ into a sum of a continuous $X$-valued semimartingale $N^{c}$ and a purely discontinuous $X$-valued semimartingale $N^{d}$, which perhaps may happen in a broader (rather than UMD) class of Banach spaces. Then for any reasonable definition of an $X$-valued semimartingale we get that $N^{c}=M^{c}+A$ for some continuous local martingale $M^{c}$ and an adapted process of (weakly) bounded variation $A$. Hence $M=N^{c}+N^{d}=$ $M^{c}+\left(N^{d}+A\right)$, where $N^{d}+A=M-M^{c}$ is a local martingale, which is purely discontinuous, so $M$ should have the Meyer-Yoeurp decomposition as well in this setting, which means that the UMD property is crucial.

\section{REFERENCES}

[1] R. Bañuelos, A. Bielaszewski, and K. Bogdan. Fourier multipliers for nonsymmetric Lévy processes. In Marcinkiewicz centenary volume, volume 95 of Banach Center Publ., pages 9-25. Polish Acad. Sci. Inst. Math., Warsaw, 2011.

[2] R. Bañuelos and K. Bogdan. Lévy processes and Fourier multipliers. J. Funct. Anal., 250(1):197-213, 2007.

[3] P. Billingsley. Probability and measure. Wiley Series in Probability and Mathematical Statistics. John Wiley \& Sons, Inc., New York, third edition, 1995. A Wiley-Interscience Publication.

[4] D.L. Burkholder. Martingale transforms. Ann. Math. Statist., 37:1494-1504, 1966.

[5] D.L. Burkholder. Distribution function inequalities for martingales. Ann. Probability, 1:19-42, 1973.

[6] D.L. Burkholder. A geometrical characterization of Banach spaces in which martingale difference sequences are unconditional. Ann. Probab., 9(6):9971011, 1981.

[7] D.L. Burkholder. Boundary value problems and sharp inequalities for martingale transforms. Ann. Probab., 12(3):647-702, 1984. 
[8] D.L. Burkholder. Sharp inequalities for martingales and stochastic integrals. Astérisque, (157-158):75-94, 1988. Colloque Paul Lévy sur les Processus Stochastiques (Palaiseau, 1987).

[9] D.L. Burkholder. Differential subordination of harmonic functions and martingales. In Harmonic analysis and partial differential equations (El Escorial, 1987), volume 1384 of Lecture Notes in Math., pages 1-23. Springer, Berlin, 1989.

[10] D.L. Burkholder. Martingales and singular integrals in Banach spaces. In Handbook of the geometry of Banach spaces, Vol. I, pages 233-269. NorthHolland, Amsterdam, 2001.

[11] D.L. Burkholder and R.F. Gundy. Extrapolation and interpolation of quasilinear operators on martingales. Acta Math., 124:249-304, 1970.

[12] G. Da Prato and J. Zabczyk. Stochastic equations in infinite dimensions, volume 152 of Encyclopedia of Mathematics and its Applications. Cambridge University Press, Cambridge, second edition, 2014.

[13] S. Dirksen. Itô isomorphisms for $L^{p}$-valued Poisson stochastic integrals. Ann. Probab., 42(6):2595-2643, 2014.

[14] S. Dirksen, J. Maas, and J.M.A.M. van Neerven. Poisson stochastic integration in Banach spaces. Electron. J. Probab., 18:No. 100, 28, 2013.

[15] S. Dirksen and I.S. Yaroslavtsev. $L^{q}$-valued Burkholder-Rosenthal inequalities and sharp estimates for stochastic integrals. arXiv:1707.00109, 2017.

[16] D.J.H. Garling. Brownian motion and UMD-spaces. In Probability and Banach spaces (Zaragoza, 1985), volume 1221 of Lecture Notes in Math., pages 36-49. Springer, Berlin, 1986.

[17] M. Girardi and L.W. Weis. Operator-valued martingale transforms and Rboundedness. Illinois J. Math., 49(2):487-516, 2005.

[18] L. Grafakos. Classical Fourier analysis, volume 249 of Graduate Texts in Mathematics. Springer, New York, third edition, 2014.

[19] R.F. Gundy. A decomposition for $L^{1}$-bounded martingales. Ann. Math. Statist., 39:134-138, 1968.

[20] I. Gyöngy. On stochastic equations with respect to semimartingales. III. Stochastics, 7(4):231-254, 1982.

[21] I. Gyöngy and N.V. Krylov. On stochastic equations with respect to semimartingales. I. Stochastics, 4(1):1-21, 1980/81.

[22] P. Hitczenko. Upper bounds for the $L_{p}$-norms of martingales. Probab. Theory Related Fields, 86(2):225-238, 1990.

[23] T.P. Hytönen, J.M.A.M. van Neerven, M.C. Veraar, and L. Weis. Analysis in Banach spaces. Vol. I. Martingales and Littlewood-Paley theory, volume 63 of Ergebnisse der Mathematik und ihrer Grenzgebiete. Springer, 2016.

[24] J. Jacod. Calcul stochastique et problèmes de martingales, volume 714 of Lecture Notes in Mathematics. Springer, Berlin, 1979.

[25] J. Jacod and A.N. Shiryaev. Limit theorems for stochastic processes, volume 288 of Grundlehren der Mathematischen Wissenschaften. Springer-Verlag, Berlin, second edition, 2003.

[26] O. Kallenberg. Foundations of modern probability. Probability and its Applications (New York). Springer-Verlag, New York, second edition, 2002.

[27] D. Kannan and V. Lakshmikantham, editors. Handbook of stochastic analysis and applications, volume 163 of Statistics: Textbooks and Monographs. Marcel 
Dekker, Inc., New York, 2002.

[28] I. Karatzas and S.E. Shreve. Brownian motion and stochastic calculus, volume 113 of Graduate Texts in Mathematics. Springer-Verlag, New York, second edition, 1991.

[29] V.M. Kruglov. On natural and predictable processes. Sankhya A, 78(1):43-51, 2016.

[30] S. Kwapień and W.A. Woyczyński. Random series and stochastic integrals: single and multiple. Probability and its Applications. Birkhäuser Boston, Inc., Boston, MA, 1992.

[31] C. Marinelli. On maximal inequalities for purely discontinuous $L_{q}$-valued martingales. arXiv:1311.7120, 2013.

[32] C. Marinelli and M. Röckner. On maximal inequalities for purely discontinuous martingales in infinite dimensions. In Séminaire de Probabilités XLVI, volume 2123 of Lecture Notes in Math., pages 293-315. Springer, 2014.

[33] T. Martí nez and J.L. Torrea. Operator-valued martingale transforms. Tohoku Math. J. (2), 52(3):449-474, 2000.

[34] T.R. McConnell. Decoupling and stochastic integration in UMD Banach spaces. Probab. Math. Statist., 10(2):283-295, 1989.

[35] M. Métivier and J. Pellaumail. Stochastic integration. Academic Press [Harcourt Brace Jovanovich, Publishers], New York-London-Toronto, Ont., 1980. Probability and Mathematical Statistics.

[36] P.-A. Meyer. Un cours sur les intégrales stochastiques. pages 245-400. Lecture Notes in Math., Vol. 511, 1976.

[37] J.M.A.M. van Neerven, M. C. Veraar, and L.W. Weis. Stochastic integration in UMD Banach spaces. Ann. Probab., 35(4):1438-1478, 2007.

[38] A.A. Novikov. Discontinuous martingales. Teor. Verojatnost. i Primemen., 20:13-28, 1975.

[39] A. Osękowski. Inequalities for dominated martingales. Bernoulli, 13(1):54-79, 2007.

[40] A. Osẹkowski. Sharp martingale and semimartingale inequalities, volume 72 of Instytut Matematyczny Polskiej Akademii Nauk. Monografie Matematyczne (New Series). Birkhäuser/Springer Basel AG, Basel, 2012.

[41] A. Osękowski and I.S. Yaroslavtsev. The Hilbert transform and orthogonal martingales in Banach spaces. arXiv:1805.03948, 2018.

[42] J. Parcet and N. Randrianantoanina. Gundy's decomposition for noncommutative martingales and applications. Proc. London Math. Soc. (3), 93(1):227-252, 2006.

[43] G. Pisier. Martingales in Banach spaces, volume 155. Cambridge University Press, 2016.

[44] D. Pollard. Convergence of stochastic processes. Springer Series in Statistics. Springer-Verlag, New York, 1984.

[45] P.E. Protter. Stochastic integration and differential equations, volume 21 of Stochastic Modelling and Applied Probability. Springer-Verlag, Berlin, 2005. Second edition. Version 2.1, Corrected third printing.

[46] H.P. Rosenthal. On the subspaces of $L^{p}(p>2)$ spanned by sequences of independent random variables. Israel J. Math., 8:273-303, 1970.

[47] J.L. Rubio de Francia. Martingale and integral transforms of Banach space valued functions. In Probability and Banach spaces (Zaragoza, 1985), volume 
1221 of Lecture Notes in Math., pages 195-222. Springer, Berlin, 1986.

[48] M.C. Veraar. Continuous local martingales and stochastic integration in UMD Banach spaces. Stochastics, 79(6):601-618, 2007.

[49] M.C. Veraar and I.S. Yaroslavtsev. Cylindrical continuous martingales and stochastic integration in infinite dimensions. Electron. J. Probab., 21:53 pp., 2016.

[50] G. Wang. Differential subordination and strong differential subordination for continuous-time martingales and related sharp inequalities. Ann. Probab., 23(2):522-551, 1995.

[51] I.S. Yaroslavtsev. Martingale decompositions and weak differential subordination in UMD Banach spaces. arXiv:1706.01731. To appear in Bernoulli, 2017.

[52] I.S. Yaroslavtsev. Even Fourier multipliers and martingale transforms in infinite dimensions. Indag. Math. (N.S.), 29(5):1290-1309, 2018.

[53] I.S. Yaroslavtsev. Fourier multipliers and weak differential subordination of martingales in UMD Banach spaces. Studia Math., 243(3):269-301, 2018.

[54] Ch. Yoeurp. Décompositions des martingales locales et formules exponentielles. In Séminaire de Probabilités, X (Seconde partie: Théorie des intégrales stochastiques, Univ. Strasbourg, Strasbourg, année universitaire 1974/1975), pages 432-480. Lecture Notes in Math., Vol. 511. Springer, Berlin, 1976.

Delft Institute of Applied Mathematics, Delft University of Technology, P.O. Box 5031, 2600 GA Delft, The Netherlands

E-mail address: I.S.Yaroslavtsev@tudelft.nl 